\title{
Contactless Testing of Circuit Interconnects
}

\author{
Abdelghani Renbi ${ }^{1} \cdot$ Jerker Delsing $^{1}$
}

Received: 29 June 2014 / Accepted: 30 April 2015 / Published online: 5 July 2015

(C) Springer Science+Business Media New York 2015

\begin{abstract}
State-of-the-art printed circuit boards (PCBs) have become extremely dense and are not fully accessible for applying physical testing solutions. Extra steps are required in the design and manufacturing process for testing advanced printed wiring boards (PWBs) with embedded passive components. This processing is further complicated by upcoming sequential build-up (SBU) technologies that provide feature sizes smaller than $10 \mu \mathrm{m}$ and that do not allow physical access for testing the interconnect between two pads. In this paper, we propose a new contactless technique for overcoming the SBU challenge for testing interconnects between embedded components. A test trace is employed as a sensor, which senses the terminations of the trace being tested. The simulation and analysis results of this study demonstrate the feasibility of this concept for application to SBU and conventional PCB/PWB interconnect testing to overcome the barriers to physical access. Robustness of the approach has been studied against packaging deviations and possible testing process variations. To ensure defect detection with feasible margins, design for testability (DfT) rules have been established for practical PCB dimensions.
\end{abstract}

Responsible Editor: H. Manhaeve

Abdelghani Renbi

Abdelghani.Renbi@ltu.se

Jerker Delsing

Jerker.Delsing@1tu.se

1 Dept of Computer Science, Electrical, \& Space Engineering, Luleå University of Technology, Luleå, Sweden
Keywords Electronics testing · DfT · Contactless testing · Interconnect testing $\cdot$ PCB testing $\cdot$ Boundary scan

\section{Introduction}

Testing electronics is vital from the points of view of quality and economics. Although a design meets its signal integrity and other requirements which ensure a proper product functionality, manufacturing testing is still necessary due to the imperfection of the assembly process which might lead to several PCB defects such as opens, shorts, bridges, missing or wrong components, etc. Electronic devices for which failure consequences are critical require $100 \%$ confidence for being defect free. Moreover, at the end-user stage, failing electronics may be detrimental to the business and the product brand [25]. In terms of manufacturing costs, it is always cheaper to detect defects at earlier stages of the production process by removing the defective part before any further integration [2] occurs; e.g., soldering expensive packaged chips on defective PWBs may badly affect the profitability of the product.

However, high frequency, portability and low-cost manufacturing continue to encourage the miniaturization of electronics. To meet miniaturization requirements, technology is shifting toward the use of high-density interconnect (HDI) boards with embedded active chips and passive components in which interconnections are made without conventional soldering, as shown in Fig. 1. Due to the above requirements, testing joints becomes challenging due to several factors, such as structure and size. The joints that connect component pads with interconnects may not allow for physical probing or visual access. Moreover, probing fragile interconnects may degrade the short- or long-term reliability of the interconnects or may even be destructive, causing 
damage immediately after testing. Although test pads are provided in the design which do not serve small feature sizes, mechanical probing induces strain due to the probe force applied, and thus, cracks in solder joints might occur or might be initiated, later inducing failure [17]. In addition, physical probing entails the costs of probe needle maintenance [18].

SBU technology represents another advanced approach for electronic assembly through which chips and passive components are embedded and that may use nonconventional soldering processes. SBU processes offer several advantages in terms of manufacturing cost, performance, product size and short-/long-term reliability, and these advantages have been thoroughly discussed in [5], [4], [6]. Generally, manufacturing cost scales with size and production volume, the economic advantage of reducing board size by embedding passive components has been demonstrated in [9]. With the SBU process, cost can be saved in board size reduction when embedding passives and bare dies. In addition, process steps are relatively few as compared to conventional assembly process which involves several soldering steps [4]. The approximate saving from moving to SBU with embedded components is not trivial to estimate as it depends on the application. After building SBU layers, as illustrated in Fig. 1, the joints of the solderless interconnections between active and passive components may be formed as buried microvias, which are not visible and are not guaranteed to reach the pads of the components. Thus, physical and visual access is not allowed for probing or visual inspection.

During the SBU process, layers of components and interconnects should be built and tested successively. This enables the early discontinuation of part production and the initiation of interconnect process checks, thus preventing time and raw material loss. This requires fast, non-powered and, preferably, non-contact test methods. Unfortunately, the current test solutions may not be feasible or constitute the best solution for the SBU layer during manufacturing due to several reasons, such as the following:

- Direct physical and visual access being nearly impossible

- Small feature size and fragility prohibiting extra pads and direct electrical probing

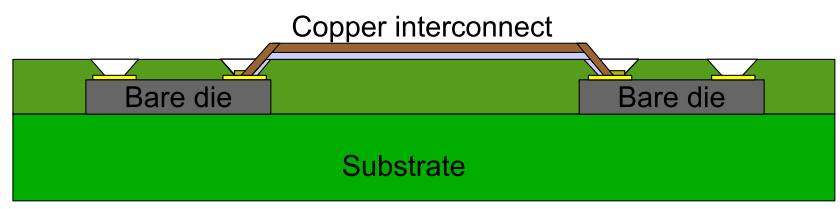

Fig. 1 Copper is deposited using methods like masked evaporation, covalent bonding metallization [16]
- The diversity of components (passive, active, ana$\log$, digital, boundary scan and non-boundary-scancompliant components)

- Layers lacking power supply lines

- The main unit not being powered during the manufacturing process.

In addition, embedded components are not necessarily designed for testability, such as in boundary-scan devices. Although testing has become an important part of the PCB process, most of the currently used testing methods are not designed for embedded chips. Moreover, a finished PWB is only tested for continuity and isolation and is only occasionally tested for its characteristic impedance. In [15], the authors presented a new solderless interconnection technique for active components, in which a daisy chain was employed to test the connections. However, how can active devices be tested in a scenario where chain-based testing is not possible? Testing embedded passive components has always required probing, where vias and/or extra active signal processing blocks are used for measurement [12].

The content of this paper can be divided in several parts, in addition to the aforesaid motivations and challenges, the next coming Section 2 continues with the aspects of the current test solutions. Sections 3 through 10 introduce the main contribution with some related work and provide the theoretical background and proof for the feasibility of the contacltess test approach, they also present simulations, verifications and analysis of the approach robustness against possible process deviations. This part also presents a demonstration of the approach feasibility on real conventional PCB with diverse components. Sections 11 through 14 deal with DfT rules that have been derived to ensure $10 \%$ as minimum margin, this to ensure defect detection for possible dimensions and realistic design cases. Within the same part, approach limitations and performance have been discussed hypothetically.

\section{Boundary-scan and Current Limitations}

Boundary-scan testing is a test method used to resolve the limited physical access problem. Generally, it is known as the IEEE 1149.1 standard and is widely accepted and used for board level testing; however, by its nature, it is limited to interconnects between digital ICs where at least one IC is standard compliant. In such scenarios, clustertesting techniques are applied to test interconnects between non-boundary-scan digital ICs and IEEE 1149.1-standardcompliant devices [21]. In many cases, extra probing and measurements are necessary to achieve $100 \%$ defect coverage, although a PCB under test has several IEEE 1149.1standard-compliant devices, e.g., analog-to-digital (ADC) 
and digital-to-analog (DAC) converter cases. Due to the diversity of PCB components used and due to limited physical access, other test solutions and standards are needed to enhance the defect coverage for interconnects to devices such as the following:

- Passive components

- Non-boundary-scan-compliant interconnects

- Mixed-signal ICs

- Open sockets

To overcome the issues that are related to the capabilities of analog measurements and to passive component testing, IEEE 1149.1 has been extended to IEEE 1149.4 [30]. However, up to now, IEEE 1149.4 has not been widely used commercially due to several reasons, such as its IC area concern; its implementation costs; its low measurement frequency bandwidth, which is approximately $1 \mathrm{MHz}$; and its higher measurement error, which may arise because of the higher impedance of silicon switches [8, 22]. With this situation, boundary-scan defect coverage remains unimproved in the same manner as coverage with IEEE 1149.1.

With the emergence of low-voltage differential signaling (LVDS), the IEEE 1149.1 standard has become ineffective for testing AC-coupled differential interconnects because it may generate false reports due to the connecting capacitors. To resolve this issue, IEEE 1149.6 has been developed to support both DC- and AC-coupled interconnects.

From a research perspective, boundary-scan standards have thus far made substantial progress in covering most of the possible test cases; however, testing interconnects to open sockets was not addressed until the birth of IEEE 1149.8.1, which addresses vacant connector testing. With this new standard, pulses are generated from a standardcompliant device to a vacant connector or IC packages where pins are inaccessible. A capacitive sensing plate technique is employed to detect opens and bridges in the device being tested by monitoring the received pulse level. However, this new standard still remains with two undetectable defects in the case of a short across a capacitor in series with an empty socket within a single line interconnect and with a short across the termination resistor in the case of AC-coupled interconnects [21, 29].

Table 1 summarizes the discussed standards with their specific features.

Built-in self-test (BIST) is another approach to test board interconnects against opens. Unfortunately the nature of SBU process and the need for sequential testing for earlier defect detection does not guarantee that a layer can be powered-on for testing. In addition, BIST might not be justified as it increases the cost in the test circuitry and might decrease the yield because of the associated hardware overhead [27]. Moreover, BIST would be needed on most of the ICs, and it could only be run when powered-on.
Table 1 Specific features of boundary-scan standards

\begin{tabular}{ll}
\hline Standard & Interconnect type \\
\hline IEEE 1149.1 & Between digital ICs \\
IEEE 1149.4 & Between mixed-signal devices \\
IEEE 1149.6 & Differential interconnects \\
IEEE 1149.8 .1 & To non-boundary-scan devices \\
& (Non-contact sensing is required) \\
\hline
\end{tabular}

\section{Contactless Test Method}

In view of the aforementioned factors and challenges, we propose a novel contactless testing approach that tests the quality of the interconnect. This approach has the potential to overcome several challenges that are faced by the current testing technology. Depending on the test strategy, it may be employed to fill the remaining gaps to enable better defect coverage. In the cases where physical access is impossible or where the previously mentioned boundary-scan standards are not applicable, this approach can potentially play a role in improving the defect coverage. In principal, direct physical probing on fragile pads will not be needed; the power supply of the unit under test as well as the diversity of the components will not continue to be problematic, either. The method is based on two broadside-coupled striplines, which are characterized in [11]. Galvanic measurement is preferred to determine the integrity of joints that connect embedded, active and passive components via an interconnect. This measurement is performed while the board under test is powered off. Figure 2 illustrates two embedded bare dies that are encapsulated by an insulator with $\varepsilon_{r}$, supported by a copper ground plane.

After connecting the bare dies together, the suitability of these connections is tested without physical probing. Figure 3 illustrates the scenario in which a test board consisting of a sensing line, an insulator and a ground plane is mounted to the unit under test and in which all of the components form a broadside-coupled stripline structure with terminated ends. After feeding the sensing line with one frequency signal, the ratio between the near and far end voltages in the test vehicle provides information on

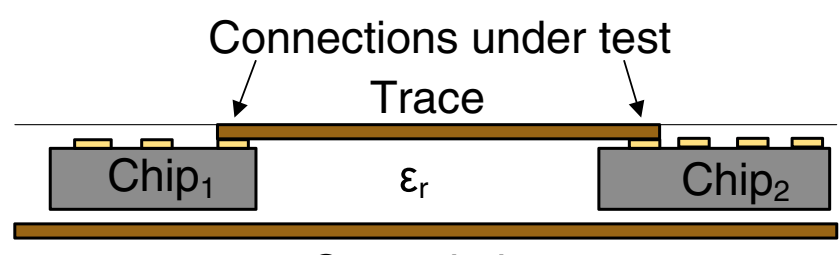

Ground plane

Fig. 2 Connection under test between two embedded active chips 


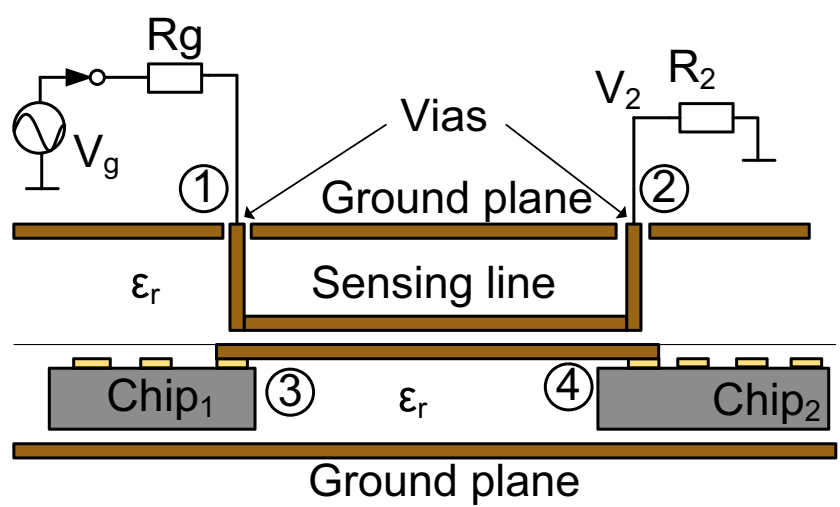

Fig. 3 Sensing line on test board forming broadside-coupled sriplines

the interconnect terminations using dual-stripline crosstalk behavior.

Mass-producing and testing multilayers may look like Fig. 4 which models a separate and demountable test vehicle that consists of a ground plane and a set of sensing lines that are designed such that they are parallel to the interconnects under test, forming a high coupling through a small thickness of the SBU insulator. Testing will be part of the SBU process; each layer is tested without probing the interconnects and without power.

In Fig. 4, the insulator is represented by a transparent material to emphasize multilayer testing as well as the shape of the sensing lines, which should follow the lines under test. While testing, the sensing lines board and the board under test must be combined in such a way that they are parallel and separated by a thin layer of insulator material. Each line from the board under test is overlapped with its image

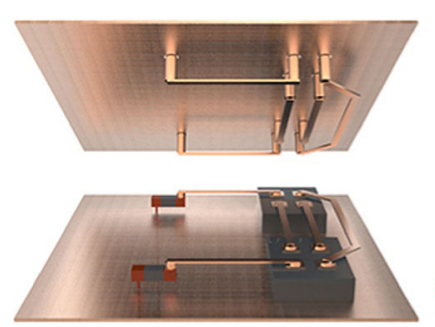

(a) First layer testing

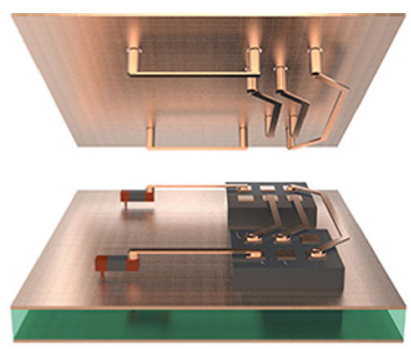

(b) Second layer testing

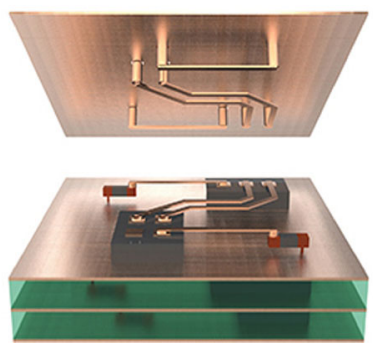

(c) Third layer testing

Fig. 4 Contactless approach to solderless SBU process testing line in the test board, forming a broadside-coupled stripline structure.

If the board under test is a conventional PCB whereby the components are not embedded, the test board must have cutouts that correspond to the surface-mounted components in the board under test. In this way, the desired structure and proximity between the sensing lines and the lines under test are formed.

\section{Related Work}

A few studies have been published on contactless testing. As discussed earlier in Section 2, in [29], the authors presented a technique for testing non-boundary-scan ICs, passive components and empty sockets by measuring the capacitive coupling on the IC packages when the IOs are driven by other boundary-scan-device signals. This technique targets conventional PCBs and relies on other boundary-scan-device signals, suggesting that PCBs are tested online.

This technique has also been extended to BGA memory packages, which cause difficult challenges due to the lack of design for test (DfT) implementations. Because of the lack of other test alternatives, memory devices may remain untested or can be time consuming due to the serialized boundary-scan access. Moreover, applying capacitive sensing to memory packages entails DfT costs for packaging and test preparation which is not straightforward. A plate with capacitance greater than $20 \mathrm{fF}$ is required to be embedded on the top of the package to the pin, and, at the least, the IEEE 1149.1 standard is supported by the memory controller and the IEEE 1149.8.1 standard is supported in the case of differential interconnects [23].

In [20], the authors introduced a non-invasive probing technique for PWB defect detection based on a capacitive measurement method. Though their approach is interesting, while scanning, the probe must be positioned mechanically, which will have a dominant effect on the test performance.

Another non-invasive test solution, which attempts to examine PCBs based on magnetic imaging, was published in [31]. Regardless of the other drawbacks that this technique might possess, the PCB must be powered on, which might not be possible within SBU process.

In addition to the board level, contactless testing has received substantial interest in terms of chip-level testing, especially with the emergence of 3D IC stacking. Throughsilicon via (TSV) technology is driving the development of contactless testing methods due to the shrinkage of size at the wafer level. The authors of [14] introduced a new approach for detecting open TSVs by adding a sensing test structure as part of DfT. This solution offers great potential 
for TSV testing; however, the testing part needs to occur in every unit under test.

In [18], the authors briefly discussed three communication methods for contactless wafer testing: Radio frequency (RF) or far-field-based communication, near-fieldbased communication and optical communication. Depending on the distance between the wafer and the test vehicle, either near- or far-field testing will be suitable for testing. In the same paper, and in [19], it is reported that a complete contactless technique has been implemented and applied to production. However, this technique employs transceivers and antennas in the devices under test, which represents the core of the approach. In addition, the defect detection mechanism is still based on boundary-scan.

To eliminate the physical access issue and to avoid pad deformation in the wafer, a capacitive-coupling-based interface has been introduced for contactless wafer testing [13]. The interface and the pads of the unit under test form a capacitor when they are separated by a distance of approximately $10 \mu \mathrm{m}$. Thus, one can probe at the interface pad in a specific range of frequencies. This solution requires the test operation of the unit under test and the subsequent virtual probing of the signal of interest. With this solution, the attenuation of the received signal depends on the separation distance, frequency and type of coupling. Inductive coupling is another alternative that can be employed for better bandwidth and for better separation flexibility.

\section{Theory, Test and Evaluation Procedure}

To analytically determine an expression that involves the impact of defects on a line under test, the model described in [11] is employed. The structure is extended to the SBU

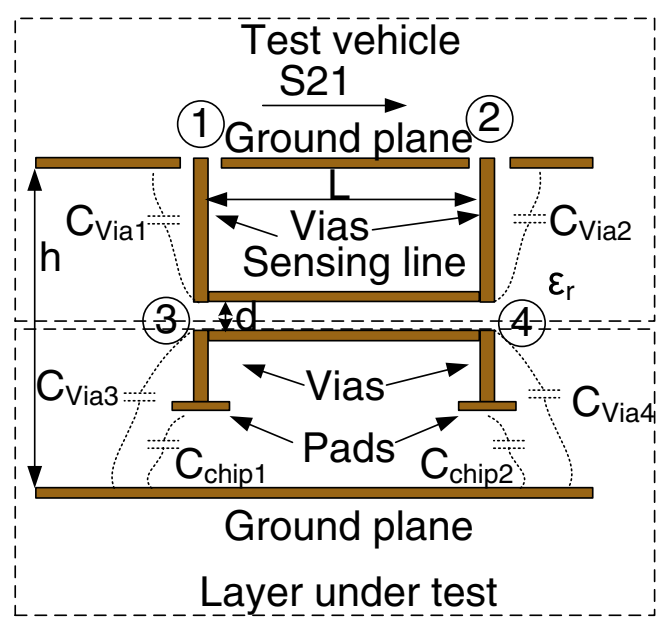

Fig. 5 Sensing line and line under test with modeled IO and joints

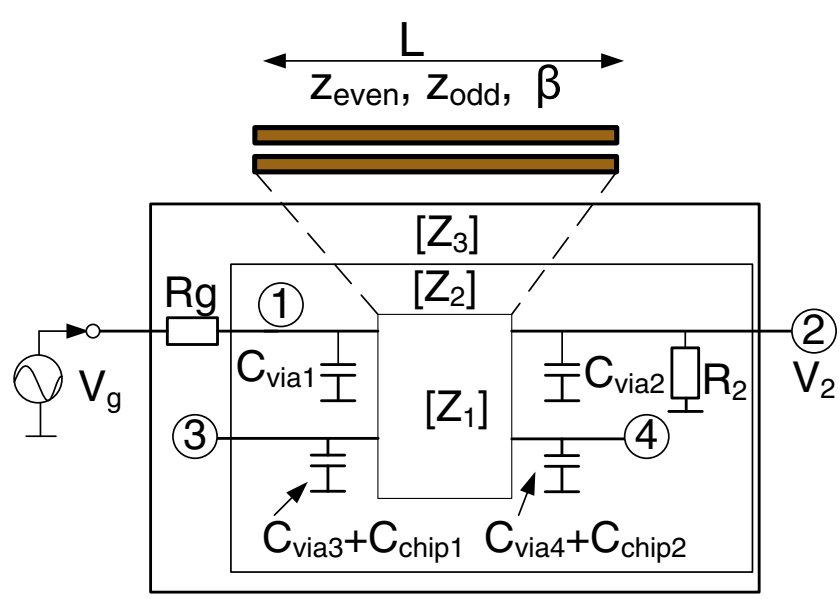

Fig. 6 Impedance network models of the terminated broadsidecoupled striplines

scenario as described by Fig. 5. A network model of Fig. 5 is presented in Fig. 6. The capacitive parts of the vias are included while the inductive parts are excluded due to the short lead lengths. The upper stripline represents the sensing line from the test vehicle, which is fed by a $50 \Omega \mathrm{RF}$ generator and terminated by a $50 \Omega$ resistor to match the results obtained with mainstream RF devices. The bottom stripline represents the line connecting the two pads of the active chips or embedded passives. By monitoring the transfer function $\frac{V_{2}}{V_{g}}$ which connects the port 1 and port 2 within a frequency range, we expect to get a response which is related to the quality of the interconnect and thus determine whether the connections are defective.

The transfer function is equal to half of the transmission coefficient $S 21$, which characterizes a network consisting of broadside-coupled striplines with their capacitive loads.

To derive the expression which relates $V_{2}$ and $V_{g}$, let $\left[Z_{1}\right]$ be the impedance matrix of the unterminated broadsidecoupled striplines being used for interconnection and sensing with length $L$. The material and geometry dimensions of the broadside-coupled striplines yield the characteristic impedances and the wave number $\beta$ where the impedances are $\left(Z_{\text {even }}\right)$ and $\left(Z_{\text {odd }}\right)$ of the even and the odd modes.

By loading the network at port 1 by $C_{V i a}$, at port 2 by $R_{2} / / C_{V i a 2}$, at port 3 by $C_{v i a 3}+C_{c h i p 1}$ and at port 4 by

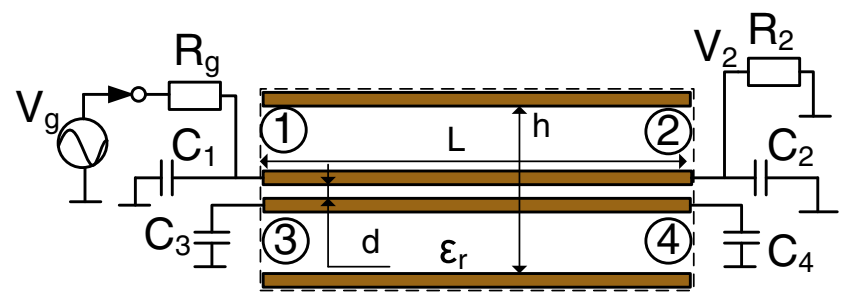

Fig. 7 Connections under test are modeled with die IO capacitances to the ground 
$C_{v i a 4}+C_{c h i p 2}$, the equivalent impedance matrix $\left[Z_{2}\right]$ will be as follows:

$\left[Z_{2}\right]=\left[\left[\begin{array}{llll}y_{11} & y_{12} & y_{13} & y_{14} \\ y_{21} & y_{22} & y_{23} & y_{24} \\ y_{31} & y_{32} & y_{33} & y_{34} \\ y_{41} & y_{42} & y_{43} & y_{44}\end{array}\right]+[\text { LoadMatrix }]\right]^{-1}$

where

$[$ LoadMatrix $]=\left[\begin{array}{llll}j C_{1} \omega & 0 & 0 & 0 \\ 0 & \frac{1}{R_{2}}+j C_{2} \omega & 0 & 0 \\ 0 & 0 & j C_{3} \omega & 0 \\ 0 & 0 & 0 & j C_{4} \omega\end{array}\right]$

Using the equation of the $\mathrm{Y}$ parameters in [11]

$\left[Z_{2}\right]=\left[Y_{2}\right]^{-1}=\left[\begin{array}{llll}a & d & b & c \\ d & e & c & b \\ b & c & f & d \\ c & b & d & g\end{array}\right]^{-1}$

where

$a=y_{11}+j C_{1} \omega=-j\left(Y_{\text {even }}+Y_{\text {odd }}\right) \frac{\cot (\beta L)}{2}+j C_{1} \omega$

$b=y_{13}=-j\left(Y_{\text {even }}-Y_{\text {odd }}\right) \frac{\cot (\beta L)}{2}$

$c=y_{14}=-j\left(Y_{\text {even }}-Y_{\text {odd }}\right) \frac{\csc (\beta L)}{2}$

$d=y_{12}=-j\left(Y_{\text {even }}+Y_{\text {odd }}\right) \frac{\csc (\beta L)}{2}$

$e=y_{11}+\frac{1}{R_{2}}+j C_{2} \omega=-j\left(Y_{\text {even }}+Y_{\text {odd }}\right) \frac{\cot (\beta L)}{2}+\frac{1}{R}+$ $j C_{2} \omega$

$f=y_{11}+j C_{3} \omega=-j\left(Y_{\text {even }}+Y_{\text {odd }}\right) \frac{\cot (\beta L)}{2}+j C_{3} \omega$

$g=y_{11}+j C_{4} \omega=-j\left(Y_{\text {even }}+Y_{\text {odd }}\right) \frac{\cot (\beta L)}{2}+j C_{4} \omega$

where $C_{1}=C_{V i a 1}, C_{2}=C_{V i a 2}, C_{3}=C_{V i a 3}+C_{C h i p 1}$,

$C_{4}=C_{v i a 4}+C_{\text {chip } 2}$ and $\omega$ is the angular frequency.

By loading the network whose impedance matrix is [ $Z_{2}$ ] by $R_{g}$ at port 1 , we complete the full transfer function:

$\frac{V_{2}}{V_{g}}=\frac{z 3_{21}}{z 3_{11}}=\frac{z 2_{21}}{R_{g}+z 2_{11}}=\frac{S 21}{2}$

where $z 3_{11}=R_{g}+z 2_{11}, z 3_{21}=z 2_{21}$ and $S 21$ is the transmission coefficient of the full network. This network consists of the broadside-coupled striplines with its capacitive loads with ports 3 and 4 discarded; otherwise, $50 \Omega$ loads are connected virtually at ports 3 and 4 while processing conversions from $\mathrm{Z}$ to $\mathrm{S}$ parameters, thus resulting in a mismatch with the structure loads. This problem occurs only during simulation if one does not discard ports 3 and 4 .

In this work an altered interconnect quality is expected to change the values of $C_{3}$ and $C_{4}$. It is thus expected that erroneous interconnect will lead to a modified value of the transfer function, thus enabling the detection of interconnect errors.

\section{Simulation Model of Test Situation}

Let us assume two bare die chips are connected using SBU approach according to Fig. 2. Figure 3 illustrates the concept of testing the interconnects using the contactless approach. After connecting the IOs of the two bare dies, which are encapsulated by an insulator with $\varepsilon_{r}$ and supported by a copper ground plane, a test board consisting of a sensing line, an insulator and a ground plane mounts to the test unit. Together, the components form a broadside-coupled stripline structure [11] with termination loads. An electrical equivalent circuit of the setup is illustrated in Fig. 7, where $C_{1}$ and $C_{2}$ model the via connections of the sensing line and $C_{3}$ and $C_{4}$ model the chip loads. The effect of the chip loads $C_{3}$ and $C_{4}$ on the voltage ratio $\frac{V_{2}}{V_{g}}$ is monitored within a frequency sweep. This ratio is equal to half of the transmission coefficient $\frac{S 21}{2}$ when $R_{g}=R_{2}=50 \Omega$ [7], which is the case when using a network analyzer.

\subsection{PEEC simulations}

To demonstrate the feasibility of the concept, one can use the analytical model described in [11]. However, for better accuracy, electromagnetic (EM) simulation can be more

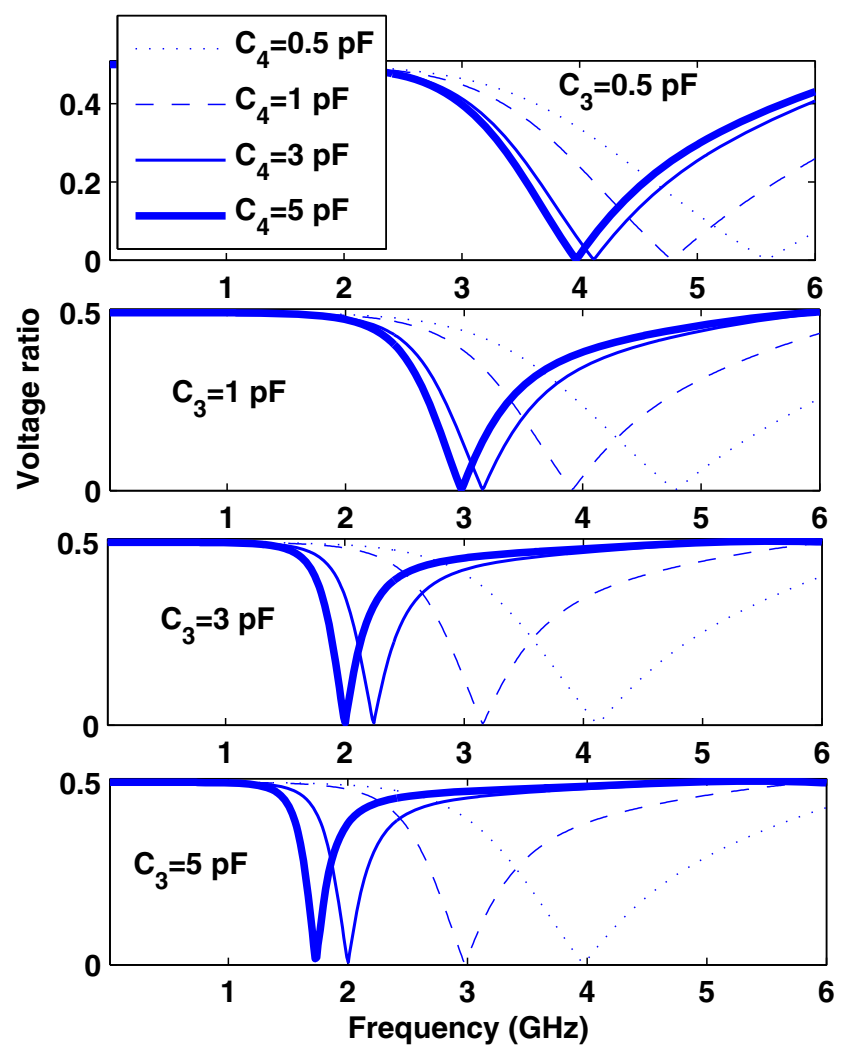

Fig. 8 Voltage ratio $\frac{V_{2}}{V_{g}}$ when 5-mm-long interconnect 


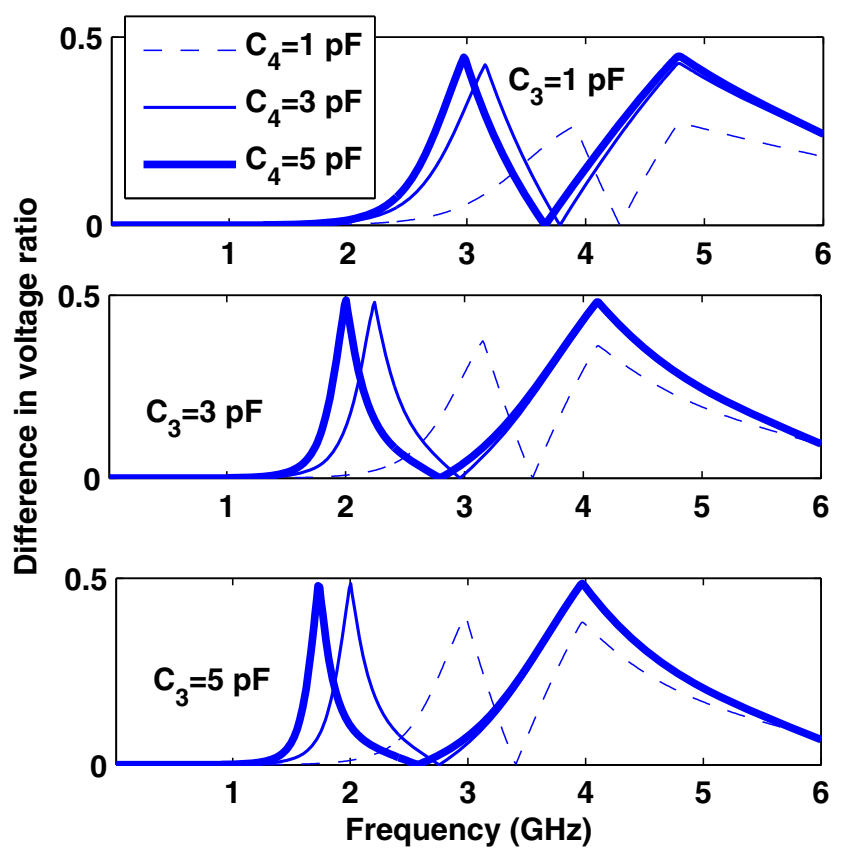

Fig. 9 Difference in voltage ratio $\frac{V_{2}}{V_{g}}$ when $0.5 \mathrm{pF}$ and other values are present in either terminations of port 3 or 4 when 5-mm-long interconnect

detailed. The scenario shown in Figs. 1 and 2 has been modeled as shown in Fig. 7. We have modeled and simulated a

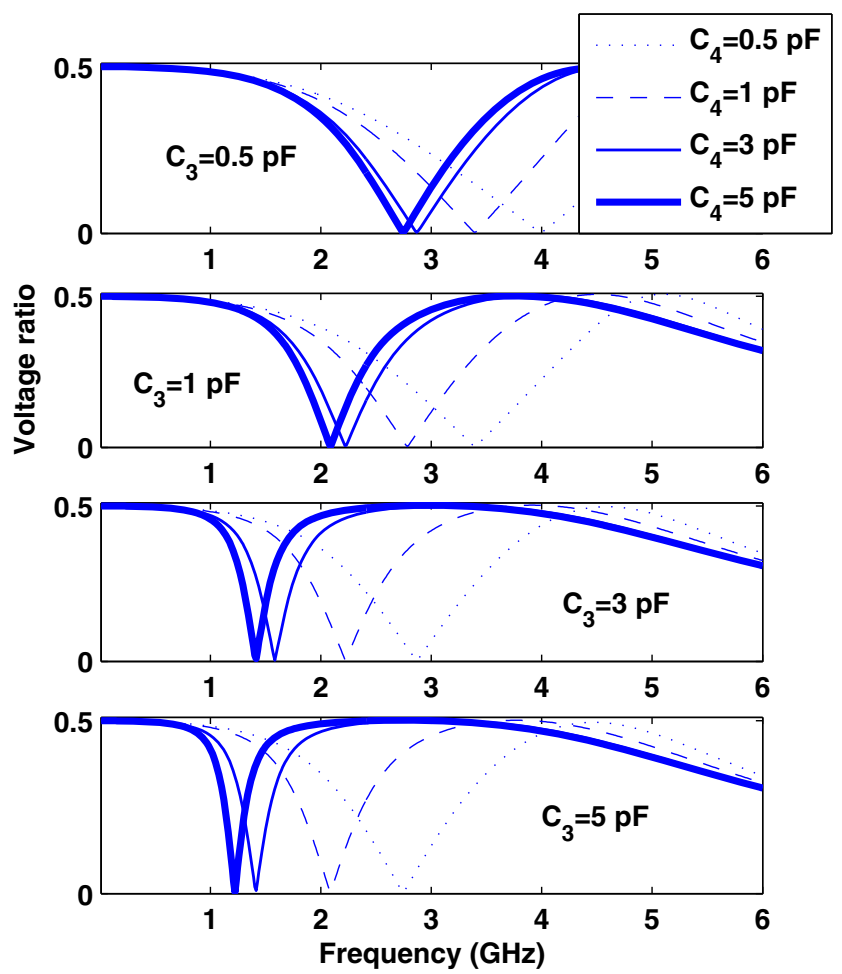

Fig. 10 Voltage ratio $\frac{V_{2}}{V_{g}}$ when 10-mm-long interconnect

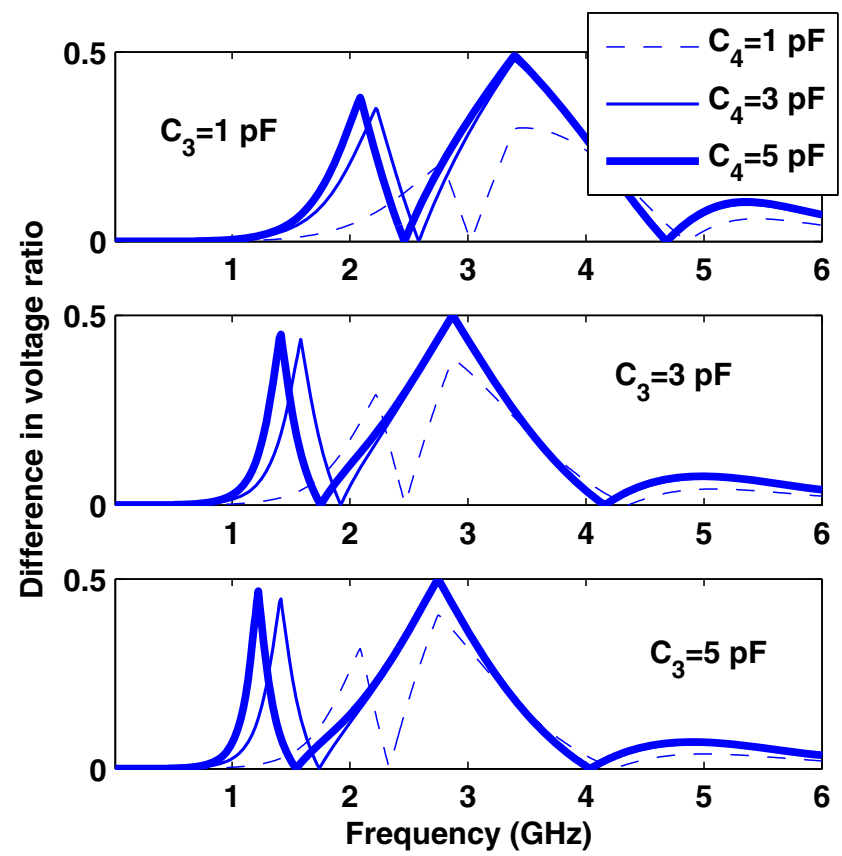

Fig. 11 Difference in voltage ratio $\frac{V_{2}}{V_{g}}$ when $0.5 \mathrm{pF}$ and other values are present in either terminations of port 3 or 4 when $10-m m$-long interconnect

number of variations to this using Multi-PEEC EM solver [24]. The parameters are varied as follows:

- Length of interconnect: 5 - $25 \mathrm{~mm}$ with $5 \mathrm{~mm}$ step.

- Load capacitance: any value from 0.5, 1, 3 and $5 \mathrm{pF}$

The selected capacitance range reflects currently manufactured ICs. According to [28], the typical IC pin capacitance to the ground, which is package-dependent, is between 2 and $8 \mathrm{pF}$. On the other hand, the trends of the IO capacitance approach $1 \mathrm{pF}$ with the package; clearly, this value will only increase with the PWB substrate. The worstcase scenario of an open defect is considered to be the lowest possible capacitance value, which in this study is considered to be $0.5 \mathrm{pF}$. In real cases, the capacitance of an open defect might be different from $0.5 \mathrm{pF}$ as it depends on how the line is connected to the chip. This difference is not problematic as the open defect is expected to decrease the load capacitance and the voltage ratio $\frac{V_{2}}{V_{g}}$ changes with the values of $C_{3}$ and $C_{4}$ as it is discussed in Section 5.

During the simulation, the partial inductances $L_{p}$, the coefficients of potential $P$ and the delay $\tau$ have been considered as PEEC simulation parameters [1]. For 5 to $25-\mathrm{mm}$ long broadside-coupled striplines, the partial resistances $R$ are too small for the structure and are worth neglecting for our purpose. Thus a correct full wave modeling of the problems has been simulated. 
The simulated structure in Fig. 7 reflects the state-ofthe-art feature size, where $100 \mu \mathrm{m}$ wide interconnects have been selected. The height $h$ includes the gap between the coupled lines, $d=50 \mu \mathrm{m}$; the thickness of the bare die, $2 \times 0.45 \mathrm{~mm}$; and the thickness of the copper layer, $2 \times 18 \mu \mathrm{m}$, which makes $0.986 \mathrm{~mm}$ in total. The insulator dielectric constant is similar to that of FR-4, i.e., $\varepsilon_{r}=4.3$.

The plots $8,10,12,14$ and 16 represent the voltage ratio response at the sensing line when both the far end and near end of the line under test are terminated by different load capacitances. According to the plots, change in the voltage ratio $\frac{V_{2}}{V_{g}}$ can be seen for frequencies close to the first resonance peak. This is a first indication of the feasibility of our approach. A significant margin is achieved at least at the first resonance frequency, as illustrated by Figs. 9, 11, 13, 15 and 17.

It should be noted that the voltage ratio does not depend on the interchangeability of $C_{3}$ and $C_{4}$; this phenomenon is proven true in Section 8 .

\subsection{Detection methods}

There are a few methods one can employ for defect detection using the above given approach. The following types of measurements highlight these methods without discussing their performance and reliability.

- First resonance frequency measurements.

- Level measurements of the transmission parameter S21 at one frequency, specifically at the resonance frequency, where a high margin is obtained between defective and correct interconnect cases.

- Signature of frequency response verification within a frequency sweep, which can be verified by using a cross-correlation approach.

The first resonance frequency refers to the first frequency where the voltage ratio goes towards zero.The voltage margin refers to the absolute difference between two voltage ratios $\left(\frac{V_{2}}{V_{g}}\right)_{\text {nominal }}$ and $\left(\frac{V_{2}}{V_{g}}\right)_{\text {measured }}$. This margin is expected to be significant when $\left(\frac{V_{2}}{V_{g}}\right)_{\text {measured }}$ corresponds to an open defect.

\subsubsection{First resonance frequency measurement}

This section discusses the use of first resonance frequency measurements to detect an open defect in an interconnect. During the test setup, reference data must be collected from a high-quality sample or from computer simulations. The reference data contain information about the first resonance
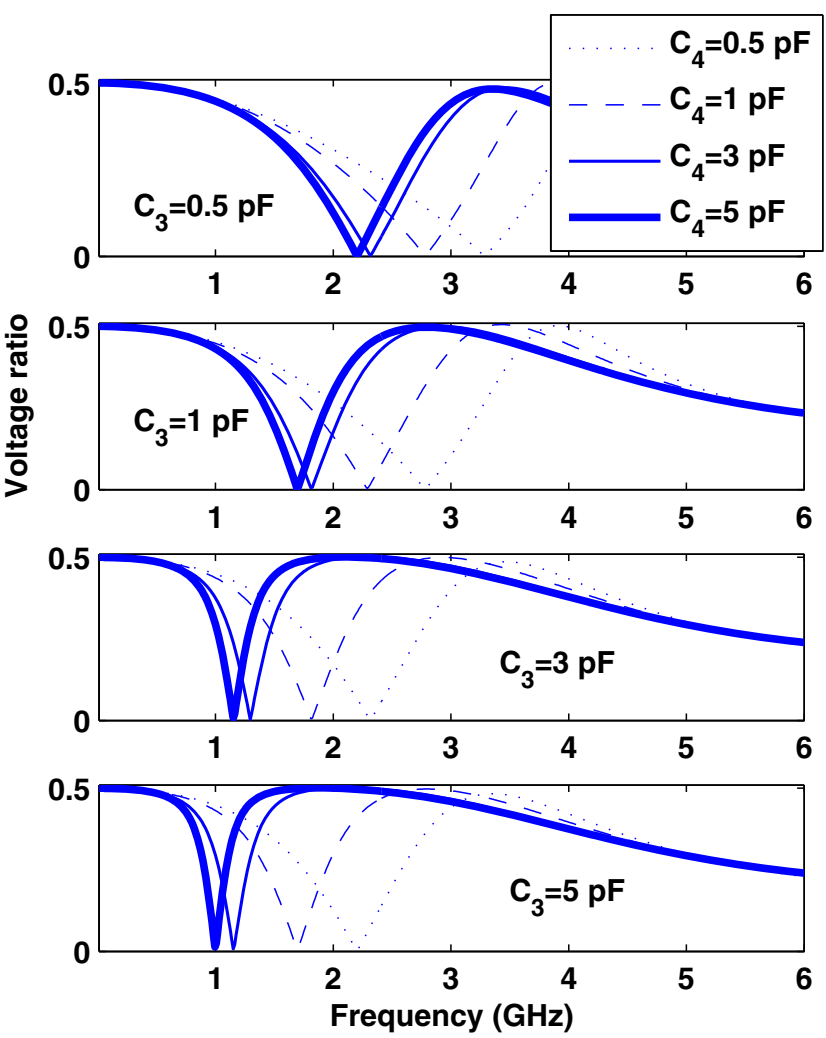

Fig. 12 Voltage ratio $\frac{V_{2}}{V_{g}}$ when $15-m m$-long interconnect
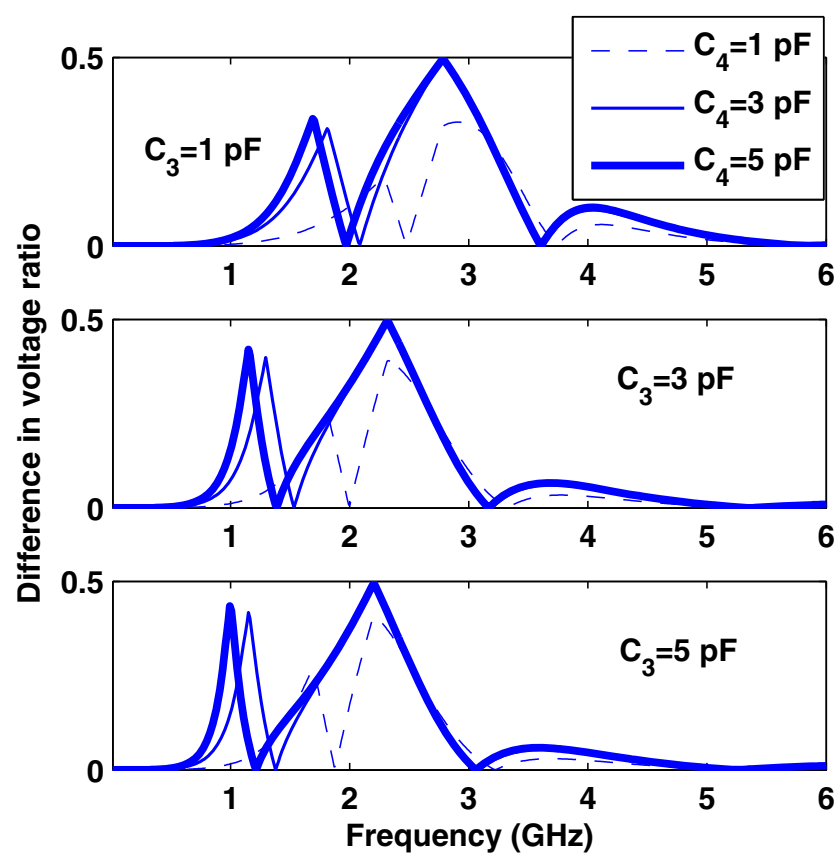

Fig. 13 Difference in voltage ratio $\frac{V_{2}}{V_{g}}$ when $0.5 \mathrm{pF}$ and other values are present in either terminations of port 3 or 4 when $15-m m$-long interconnect 
frequency of the voltage ratio of each interconnect in question for a given termination pair of capacitances. Figure 18 shows the first resonance frequencies for terminations between 0.5 and $5 \mathrm{pF}$ and for interconnects that are 5 to $25-\mathrm{mm}$-long. This resonance frequency varies between 0.77 and $5.59 \mathrm{GHz}$.

\subsubsection{Margin in voltage ratio}

For a level testing approach using one frequency, it is essential that the selected test frequency should lead to a significant margin in the voltage ratio. According to the simulation results, the first resonance frequency is the best frequency that leads to a high margin in the voltage ratio between defective and error-free interconnects. Figure 19 shows the margin in the voltage ratio at the first resonance frequency. The achieved margins are between 30 and $97 \%$ when testing interconnects that are 5 to $25-\mathrm{mm}$-long and loaded with 1 to $5 \mathrm{pF}$ for an open defect with a capacitance of $0.5 \mathrm{pF}$. This can be clearly seen in the voltage ratio difference plots in Figs. 9, 11, 13, 15 and 17. For an error free-interconnect, the level goes towards zero at the first
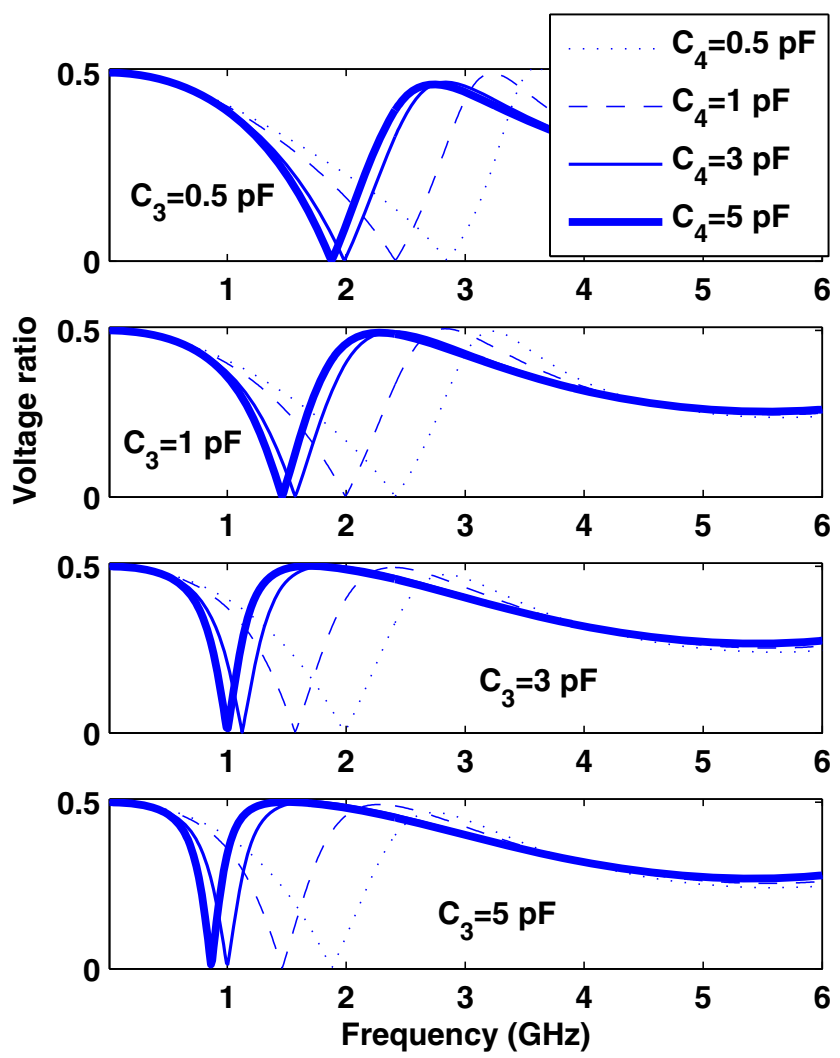

Fig. 14 Voltage ratio $\frac{V_{2}}{V_{g}}$ when 20-mm-long interconnect

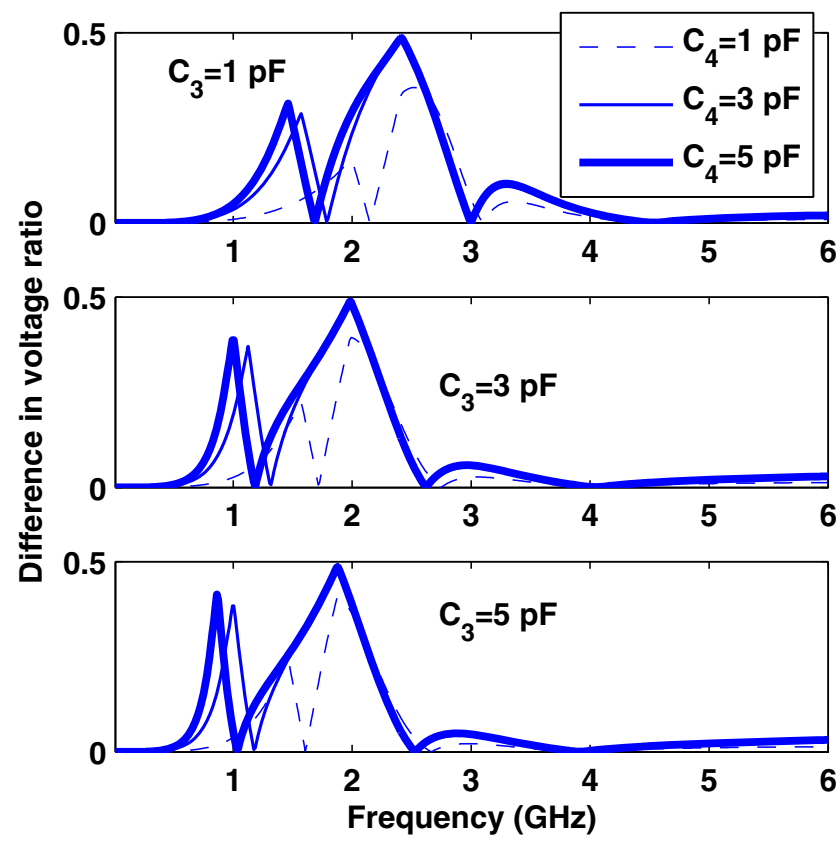

Fig. 15 Difference in voltage ratio $\frac{V_{2}}{V_{g}}$ when $0.5 \mathrm{pF}$ and other values are present in either terminations of port 3 or 4 when $20-m m$-long interconnect
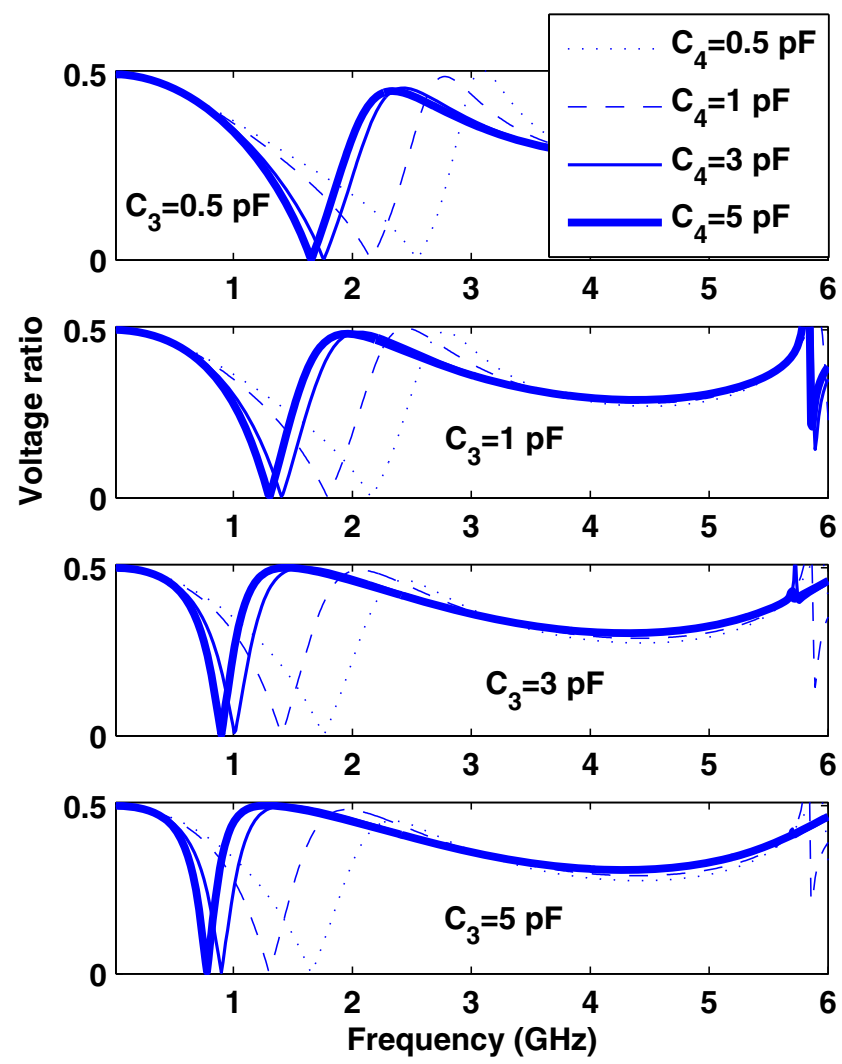

Fig. 16 Voltage ratio $\frac{V_{2}}{V_{g}}$ when 25- $m m$-long interconnect 


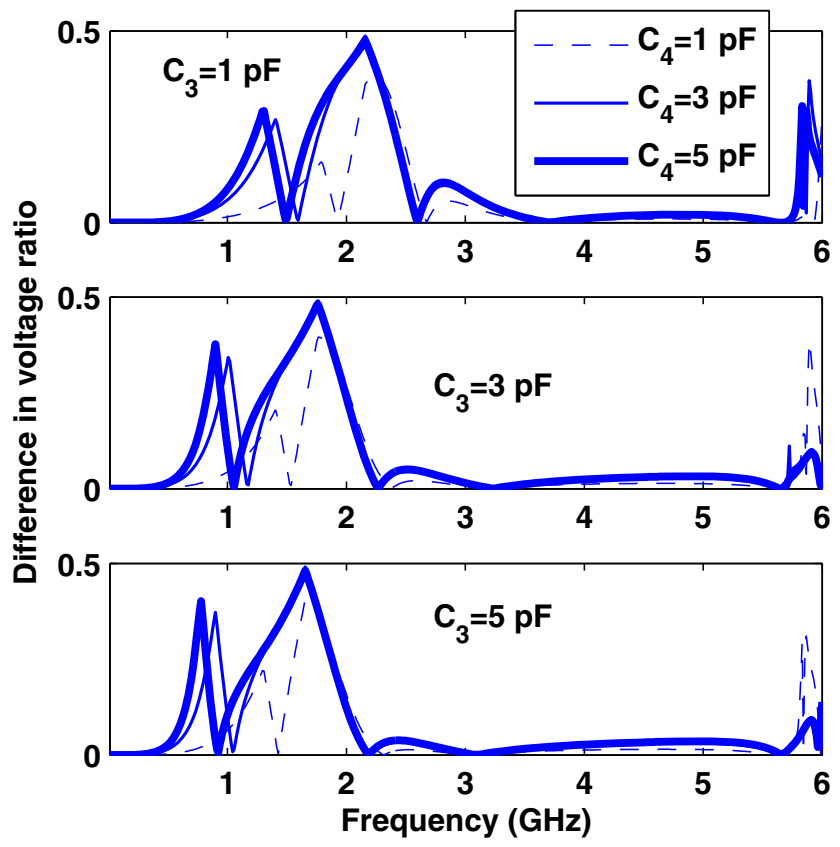

Fig. 17 Difference in voltage ratio $\frac{V_{2}}{V_{g}}$ when $0.5 \mathrm{pF}$ and other values are present in either terminations of port 3 or 4 when $25-m m$-long interconnect

resonance frequency, otherwise it is around $\frac{1}{2}$. The significance of the margin indicates that the proposed method shows good potential for detecting open defects in most cases.

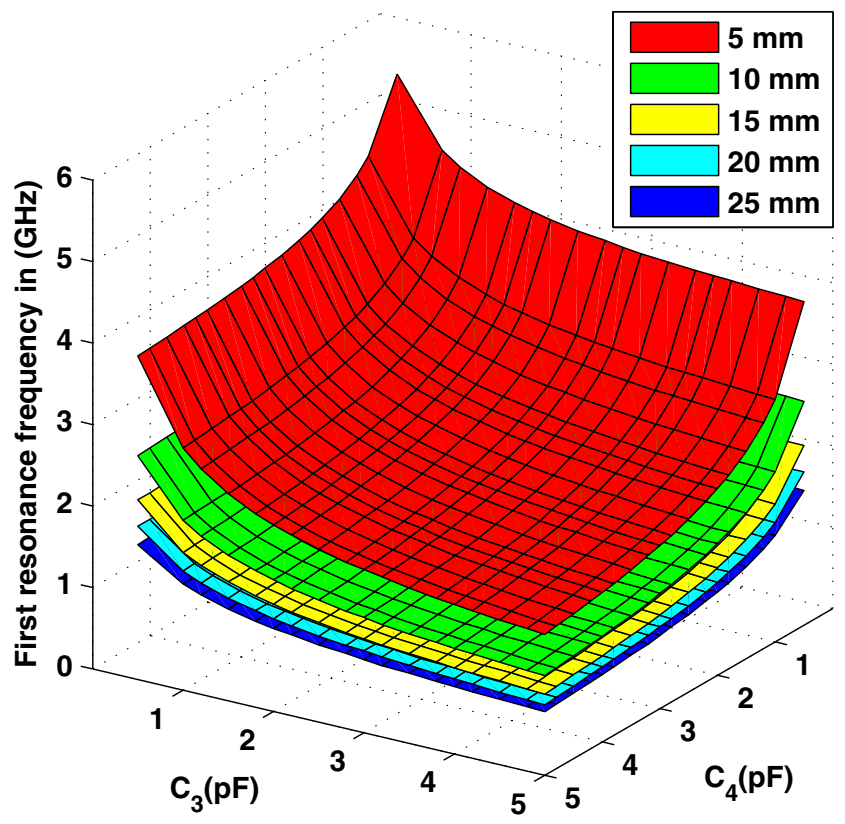

Fig. 18 Test frequency versus $C_{3}$ and $C_{4}$ for different interconnect lengths

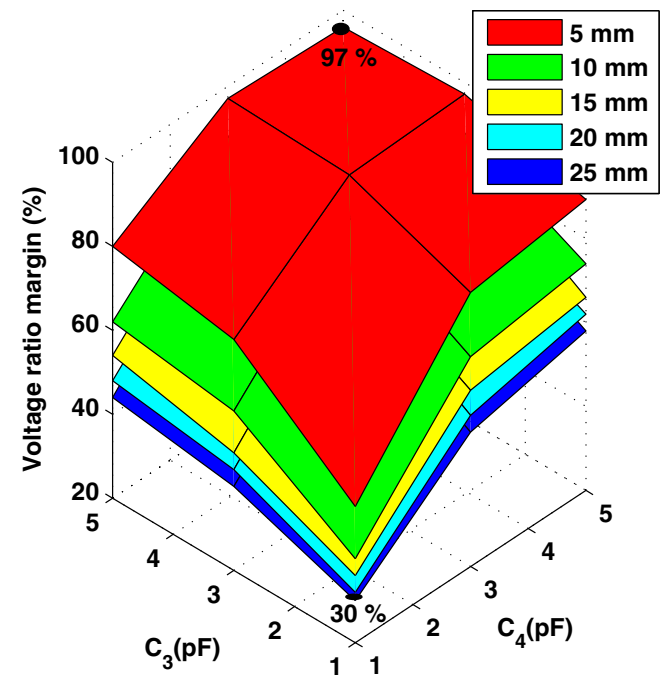

Fig. 19 Ratio margin at the first resonance frequency

\section{Experimental Verification}

\subsection{Simulated structure and prototype}

To verify the approach experimentally, the SBU structure has been prototyped on a normal PCB using SMD capacitors as chip loads. Figure 20 illustrates the structure as it appears along two views. The PCB features two broadside striplines, which are connected to the top and bottom layers

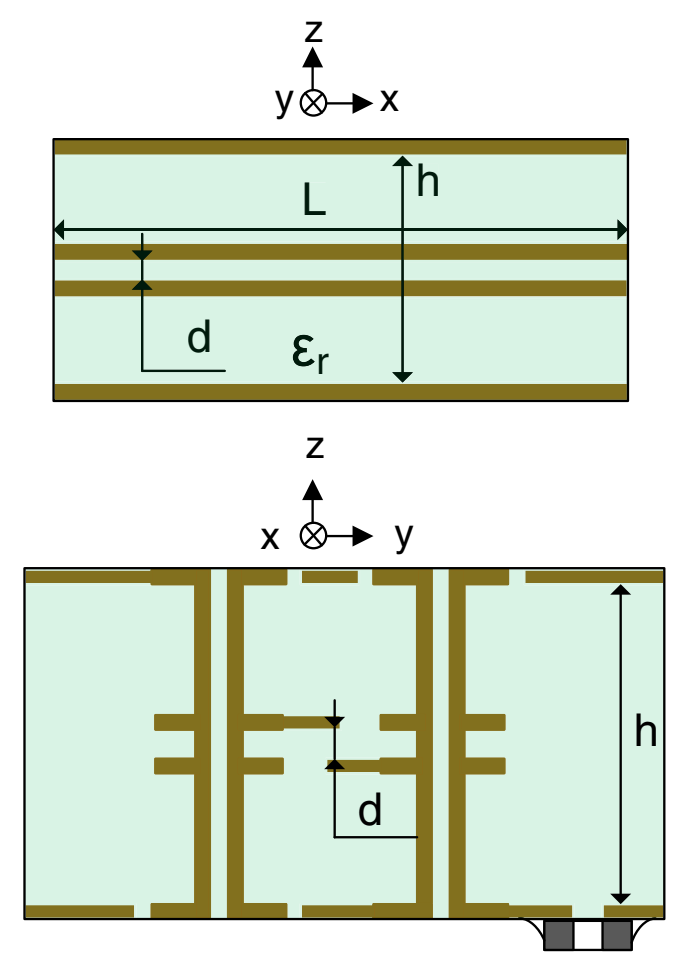

Fig. 20 SBU prototyped on conventional PCB 
through $300 \mu \mathrm{m}$ wide vias. The ground planes are separated by a distance $h=0.986 \mathrm{~mm}$, which includes the gap between the coupled lines, $d=50 \mu \mathrm{m}$; the gaps between the lines and the ground planes, $2 \times 0.45 \mathrm{~mm}$; and the thickness of the copper layer, $2 \times 18 \mu \mathrm{m}$, where FR-4 with $\varepsilon_{r}=4.3$ is used. The simulation and measurement cases involved the combination pairs $(2.2,2.2) \mathrm{pF},(2.2 \mathrm{pF}$, open $)$, $(4.7,4.7) \mathrm{pF},(4.7 \mathrm{pF}$, open $),(6.8,6.8) \mathrm{pF}$ and $(6.8 \mathrm{pF}$, open $)$ as $\left(C_{C h i p 1}, C_{C h i p 2}\right)$.

\subsection{PEEC simulations versus measurements}

The plots 21 to 25 represent the simulated and measured transfer functions at the sensing line when both the far and near ends of the line under test are terminated by a few different combination pairs of capacitance in the set $\{2.2,4.7,6.8$, open $\} \mathrm{pF}$, which covers the typical range of IC capacitance, according to [28]. Based on the plots, a significant voltage ratio margin can be achieved when sensing both ends of the trace in one pass. A significant margin is achieved at least at the first resonance frequency, as stated in Section 6.1. Thi $s$ resonance frequency is difficult to determine analytically by examining the first inflection point when solving $\left[\frac{z 2_{21}}{R_{g}+z 2_{11}}\right]^{\prime}=0$; the main reason for this complication is that the ratio involves the inverse matrix of $Y_{2}$, which makes it dense and difficult to handle. Solving for this resonance frequency through a simulation is the key to determining the test frequency, which allows for the identification of the open termination with the highest probability of being equivalent to some values in the interval $] 0,0.5] \mathrm{pF}$.
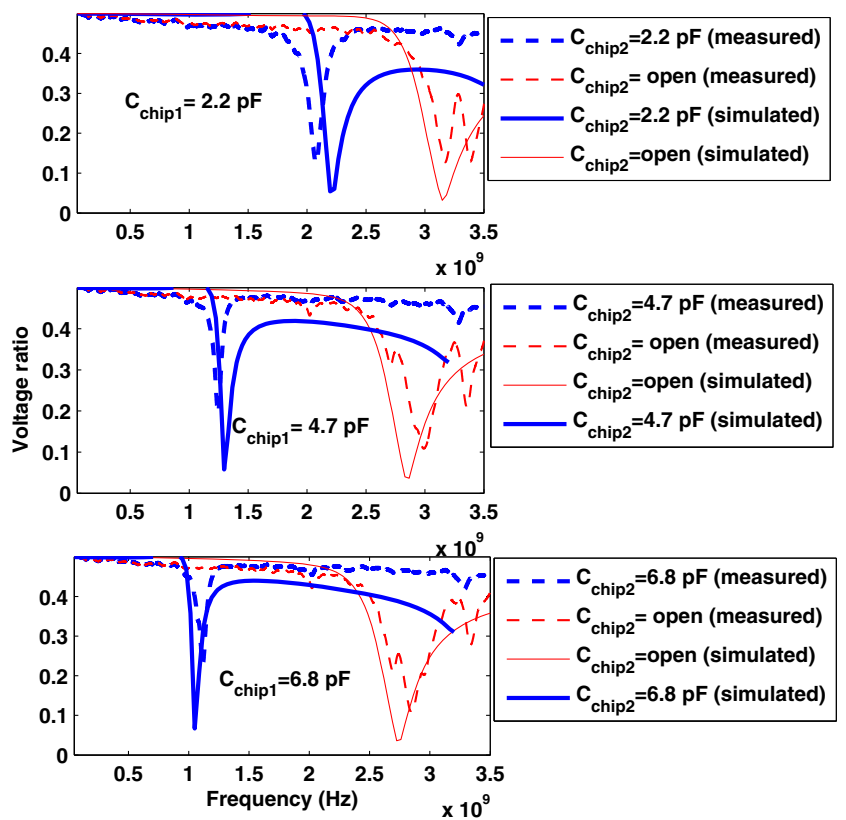

Fig. 21 Voltage ratio when line length is $5 \mathrm{~mm}$
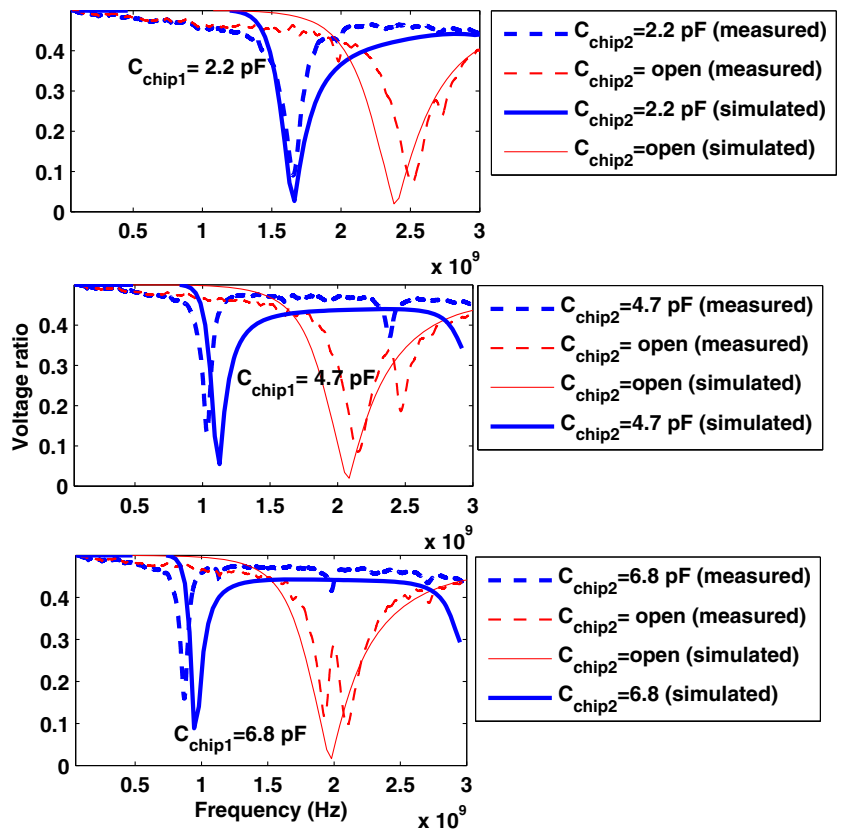

Fig. 22 Voltage ratio when line length is $10 \mathrm{~mm}$

Based on Figs. 21 to 25, the simulation results agree with the measurements, especially with respect to the shape of the curves of the voltage ratio as well how the first resonance frequency $f_{0}$ shifts with the interconnect terminations. Tables 2 to 6 have been compiled from the measurements and simulations results to compute the margin of $f_{0}$ when the interconnect under test is error-free and when it is defective. The error-free interconnect indicates that it is terminated by a proper $\left(C_{c h i p 1}, C_{c h i p 2}\right)$, where:
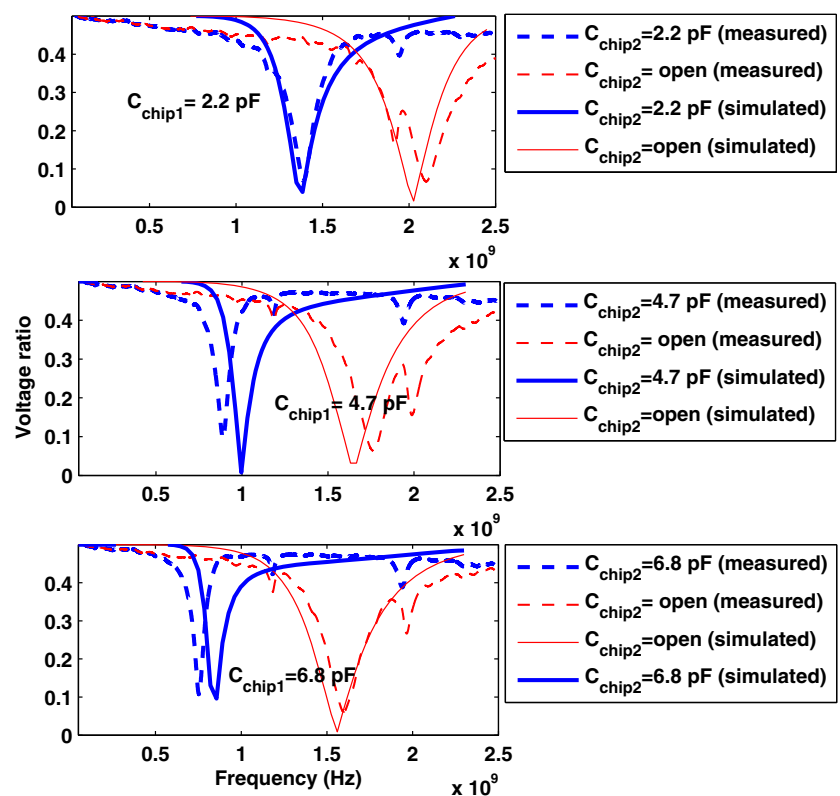

Fig. 23 Voltage ratio when line length is $15 \mathrm{~mm}$ 

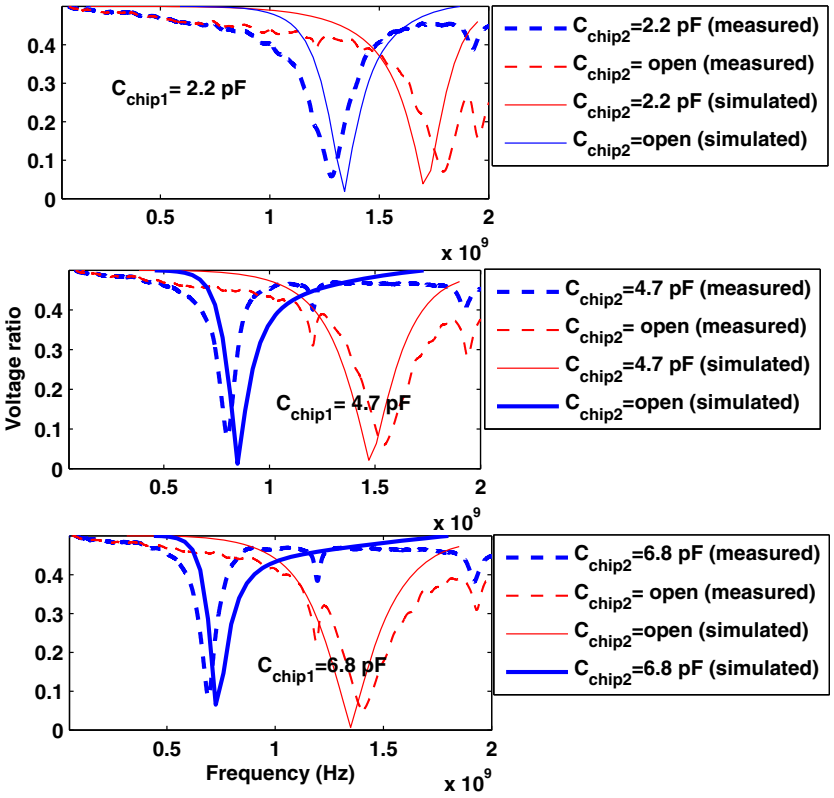

Fig. 24 Voltage ratio when line length is $20 \mathrm{~mm}$

$C_{\text {chip } 1}=C_{\text {chip } 2} \in\{2.2,4.7,6.8\} \mathrm{pF}$, whereby it can cover a realistic range of interconnect terminations. The defective interconnect indicates that $C_{c h i p 2}$ is replaced by an open, and thus, the physical value of $C_{c h i p 2}$ is approximately the via capacitance $C_{v i a 4}$.

\subsection{Margin in resonance frequency}

In this section, instead of focusing on the margin in the voltage ratio, the difference in resonance frequencies is taken as the quantity of detecting open defects or an abnormal
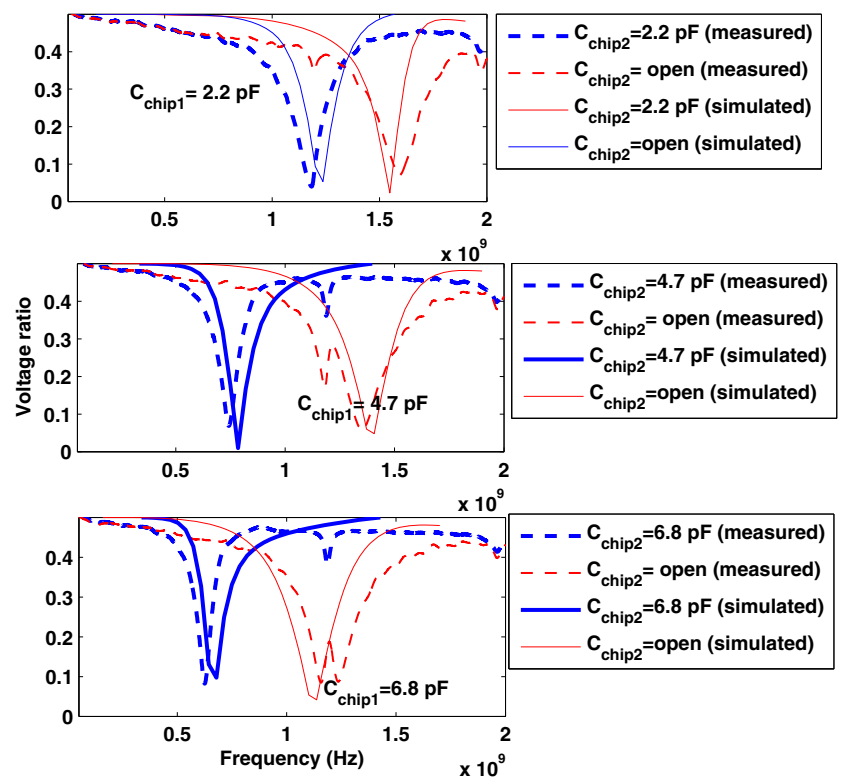

Fig. 25 Voltage ratio when line length is $25 \mathrm{~mm}$ termination in the interconnect, based on Fig. 26, which is based on the measured values collected in Tables 2 to 6 . The margin $\Delta f_{0}$ between two frequency values of $f_{0}$ is quite significant for detecting defects; this margin varies between approximately 0.41 and $1.75 \mathrm{GHz}$, i.e., between 35 and $154 \%$ of the original value. Furthermore, it is observed that the shorter the line is, the higher the margin that we obtain.

\section{Locating Defects}

Locating defects might not be useful for one unit under test. Once a unit is identified to be defective, it most likely needs to be scrapped. However, for process learning, it is useful to locate defects. By locating defects, the manufacturer may gain more inputs for root cause analysis when a process is failing repetitively and in the same joints. Thus, this section examines how the test vehicle will locate a defect when possible.

Let $T_{1}$ and $T_{2}$ be two voltage ratios, where $T_{2}$ is computed when interchanging the capacitors $C_{3}$ and $C_{4}$. Factorizing the subtraction of $T_{1}-T_{2}$ leads to the following:

$$
\begin{aligned}
f(\omega) \quad \times & \left(Y_{\text {odd }}-Y_{\text {even }}\right)^{2} R_{2}\left(C_{3}-C_{4}\right) \omega\left[R_{2} R_{g} \omega\left(C_{2}-C_{1}\right)\right. \\
& \left.+j\left(R_{2}-R_{g}\right)\right]
\end{aligned}
$$

Based on the solutions of $T_{1}-T_{2}=0$ and knowing that $f(\omega)$ is another factor function whose null condition is independent of $C_{1}, C_{2}, R_{g}$ and $R_{2}$, the two voltage ratios $T_{1}$ and $T_{2}$ are equal when $C_{1}=C_{2}$ and $R_{g}=R_{2}$. In this case, the voltage ratio is independent of the interchangeability of $C_{3}$ and $C_{4}$. This finding is in agreement with the results of our simulation cases, as indicted by the symmetry of the surface illustrated in Fig. 18. This situation is interpreted as a case in which an open defect cannot be located when testing an interconnect that is terminated by two identical loads. However, the following question arises: How does one locate a defect when testing an interconnect between two different loads?

According to Fig. 18, which has been produced using the PEEC simulations, the resonance frequency increases when $C_{3}$ and/or $C_{4}$ decrease(s). This fact can be proven analytically but that proof, because of the complexity of the analytical equation, is left out. For a given combination of $C_{3}$ and $C_{4}$, there exists a resonance frequency $f_{0}$ that is well determined and that is expected to be measured for an error-free interconnect. Let $f_{m}$ be the resonance frequency measured when testing for an interconnect between $C_{3}$ and $C_{4}$. Open joints between these two capacitors will always lead to a resonance frequency $f_{m}$ that is greater than $f_{0}$. Depending on the surface side that this frequency occurs on, the test equipment can determine the location of the open defect, which requires that the surface data be 
Table 2 Measured and simulated first resonance frequency $(\mathrm{GHz})$ in the voltage ratio in case of error-free and defective interconnect when length is $5 \mathrm{~mm}$

\begin{tabular}{|c|c|c|c|c|c|c|}
\hline & \multicolumn{6}{|c|}{$C_{\text {chip } 1}$ and $C_{\text {chip } 2}$ combinations } \\
\hline & $(2.2 \mathrm{pF}, 2.2 \mathrm{pF})$ & (2.2 pF, open) & $(4.7 \mathrm{pF}, 4.7 \mathrm{pF})$ & (4.7 pF, open) & $(6.8 \mathrm{pF}, 6.8 \mathrm{pF})$ & (6.8 pF, open) \\
\hline$f_{0}$ (measured) & 2.19 & 3.14 & 1.29 & 2.86 & 1.05 & 2.72 \\
\hline$\Delta f_{0}$ & 0.95 & & 1.57 & & 1.67 & \\
\hline$f_{0(\text { simulated })}$ & 2.07 & 3.17 & 1.24 & 2.99 & 1.11 & 2.84 \\
\hline$\Delta f_{0}$ & 1.1 & & 1.75 & & 1.73 & \\
\hline
\end{tabular}

Table 3 Measured and simulated first resonance frequency $(\mathrm{GHz})$ in the voltage ratio in case of error-free and defective interconnect when length is $10 \mathrm{~mm}$

\begin{tabular}{|c|c|c|c|c|c|c|}
\hline & \multicolumn{6}{|c|}{$C_{\text {chip } 1}$ and $C_{\text {chip } 2}$ combinations } \\
\hline & $(2.2 \mathrm{pF}, 2.2 \mathrm{pF})$ & (2.2 $\mathrm{pF}$, open) & $(4.7 \mathrm{pF}, 4.7 \mathrm{pF})$ & (4.7 pF, open) & $(6.8 \mathrm{pF}, 6.8 \mathrm{pF})$ & (6.8 pF, open) \\
\hline$f_{0}($ measured $)$ & 1.66 & 2.38 & 1.12 & 2.08 & 0.94 & 1.98 \\
\hline$\Delta f_{0}$ & 0.72 & & 0.96 & & 1.04 & \\
\hline$f_{0}($ simulated $)$ & 1.65 & 2.51 & 1.03 & 2.15 & 0.87 & 2.09 \\
\hline$\Delta f_{0}$ & 0.86 & & 1.12 & & 1.22 & \\
\hline
\end{tabular}

Table 4 Measured and simulated first resonance frequency $(\mathrm{GHz})$ in the voltage ratio in case of error-free and defective interconnect when length is $15 \mathrm{~mm}$

\begin{tabular}{lllllll}
\hline \multicolumn{7}{l}{$C_{\text {chip } 1}$ and $C_{\text {chip } 2}$ combinations } \\
\cline { 2 - 7 } & $(2.2 \mathrm{pF}, 2.2 \mathrm{pF})$ & $(2.2 \mathrm{pF}$, open $)$ & $(4.7 \mathrm{pF}, 4.7 \mathrm{pF})$ & $(4.7 \mathrm{pF}$, open $)$ & $(6.8 \mathrm{pF}, 6.8 \mathrm{pF})$ & $(6.8 \mathrm{pF}, \mathrm{open})$ \\
\cline { 2 - 7 }$f_{0}($ measured $)$ & 1.38 & 2.02 & 0.99 & 1.66 & 0.85 & 1.56 \\
$\Delta f_{0}$ & 0.64 & & 0.67 & 1.75 & 0.71 & 0.75 \\
$f_{0}($ simulated $)$ & 1.39 & 2.01 & 0.88 & & 0.84 & 1.59 \\
$\Delta f_{0}$ & 0.62 & & 0.87 & & \\
\hline
\end{tabular}

Table 5 Measured and simulated first resonance frequency $(\mathrm{GHz})$ in the voltage ratio in case of error-free and defective interconnect when length is $20 \mathrm{~mm}$

\begin{tabular}{lllllll}
\hline \multicolumn{7}{l}{$C_{\text {chip } 1}$ and $C_{\text {chip } 2}$ combinations } \\
\cline { 2 - 7 } & $(2.2 \mathrm{pF}, 2.2 \mathrm{pF})$ & $(2.2 \mathrm{pF}$, open $)$ & $(4.7 \mathrm{pF}, 4.7 \mathrm{pF})$ & $(4.7 \mathrm{pF}$, open $)$ & $(6.8 \mathrm{pF}, 6.8 \mathrm{pF})$ & $(6.8 \mathrm{pF}, \mathrm{open})$ \\
\cline { 2 - 7 }$f_{0}$ (measured $)$ & 1.34 & 1.69 & 0.84 & 1.47 & 0.72 & 1.34 \\
$\Delta f_{0}$ & 0.35 & & 0.63 & & 0.62 & 0.69 \\
$f_{0}($ simulated $)$ & 1.28 & 1.79 & 0.8 & 1.54 & 0.71 & 1.4 \\
$\Delta f_{0}$ & 0.51 & & 0.74 & & & \\
\hline
\end{tabular}


Table 6 Measured and simulated first resonance frequency $(\mathrm{GHz})$ in the voltage ratio in case of error-free and defective interconnect when length is $25 \mathrm{~mm}$

\begin{tabular}{lllllll}
\hline \multicolumn{7}{l}{$C_{\text {chip } 1}$ and $C_{\text {chip } 2}$ combinations } \\
\cline { 2 - 7 } & $(2.2 \mathrm{pF}, 2.2 \mathrm{pF})$ & $(2.2 \mathrm{pF}, \mathrm{open})$ & $(4.7 \mathrm{pF}, 4.7 \mathrm{pF})$ & $(4.7 \mathrm{pF}$, open $)$ & $(6.8 \mathrm{pF}, 6.8 \mathrm{pF})$ & $(6.8 \mathrm{pF}, \mathrm{open})$ \\
\cline { 2 - 7 }$f_{0}$ (measured $)$ & 1.23 & 1.54 & 0.78 & 1.4 & 0.67 & 1.13 \\
$\Delta f_{0}$ & 0.31 & & 0.62 & & 0.46 & 0.62 \\
$f_{0}($ simulated $)$ & 1.18 & 1.59 & 0.74 & 1.37 & 0.53 & 1.15 \\
$\Delta f_{0}$ & 0.41 & & 0.63 & & & \\
\hline
\end{tabular}

stored and compared to $f_{m}$. Figure 18 illustrates the surface of the resonance frequencies versus the load capacitors $C_{3}$ and $C_{4}$; the frequencies that fall on the upper edges of the surface are those that occur in the case of open defects, assuming that the lowest capacitor value represents an open defect or via capacitance, indicated by $C_{v i a 3}$ and $C_{v i a 4}$ in Fig. 5. However, the remaining frequencies will correspond to error-free joints, whose values depend on the connected load capacitors, which are indicated by $C_{\text {chip } 1}$ and $C_{\text {chip } 2}$ in Fig. 5. The right upper edge of the surface represents the frequencies to be detected when open defects occur on port 4 . In contrast, the left upper edge of the surface represents the frequencies to be detected when open defects occur on the port 3 side. The highest frequency will occur when open defects are formed on both sides of the interconnect.

\section{Process Deviations on Method Robustness}

In this section, we will examine the robustness of the proposed method against the compounded process deviations. Manufacturing process deviations will lead to IO

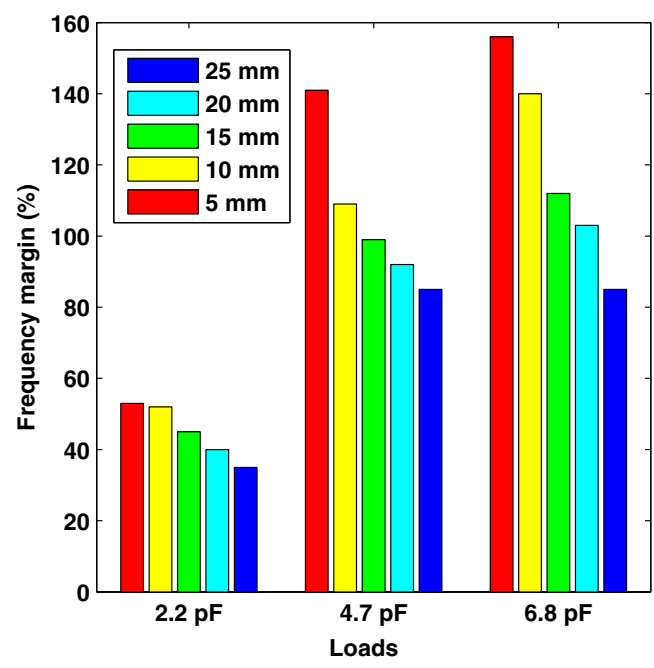

Fig. 26 First resona nce frequency margin between an error-free and defective interconnect capacitance deviations, and thus, one can speculate a shift in the resonance frequency due to packaging deviations that might lead to false alarms. In the manufacturing of electronics, the nominal value of a quantity is most often the target; therefore, we assume that the deviations of the IO capacitance are characterized by a normal distribution.

\subsection{Packaging process deviations on resonance frequency}

Let $f_{0}$ be the nominal value of the frequency of the voltage ratio. $C_{\text {chip } 1}$ and $C_{\text {chip } 2}$ are the nominal values of the capacitances representing the connected IO of the chips. After running Monte Carlo simulations for $N=50000$ samples of 861 pairs of $\left(C_{c h i p 1}, C_{c h i p 2}\right)$, which can be formed by any value from the set:

$\{1,1.1,1.2,1.3, \ldots, 4.7,4.8,4.9,5\} \mathrm{pF}$, according to Figs. 27 and 28, one can conclude that a 10\% standard deviation in and $C_{\text {chip } 2}$ leads to a small $\sigma_{f_{0}}$, whereas it is $2 \% \leq$ $\sigma_{f_{0}} \leq 5 \%$ on average. Moreover, a $20 \%$ standard deviation in $C_{\text {chip } 1}$ and $C_{\text {chip } 2}$ leads to $4 \% \leq \sigma_{f_{0}} \leq 11 \%$ on average. Generally, the length of the interconnect has almost no effect on $\sigma_{f_{0}}$.

\subsection{Probability of false alarms}

By knowing the standard deviation of the resonance frequency $\sigma_{f_{0}}$ and its mean, for a given pair of $\left(C_{c h i p 1}, C_{c h i p 2}\right)$, one can compute the resonance frequency when the smallest termination is replaced by an open termination for a worst-case scenario, i.e., when the smallest value of $C_{\text {chip } 1}$ and $C_{\text {chip } 2}$ is replaced by an open termination, its equivalent capacitance is $0.5 \mathrm{pF}$, as discussed previously. By knowing the resonance frequency $f_{0_{\text {open }}}$ in the case of an open defect, as shown in Fig. 29, one can determine the probability of reaching this frequency due to the deviation in the packaging process affecting $C_{\text {chip } 1}$ and $C_{\text {chip2 }}$. The probability is $1-\Phi\left(f_{0_{\text {open }}}\right)$, where

$\Phi\left(f_{0_{\text {open }}}\right)=\frac{1}{\sigma_{f_{0}} \sqrt{2 \pi}} \int_{-\infty}^{f_{0_{\text {open }}}} e^{-\frac{\left(f_{0}-\sigma_{f_{0}}\right)^{2}}{2 \sigma_{f_{0}}^{2}}} d f_{0}$ 


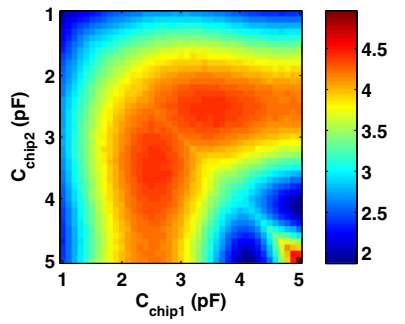

(a) $\mathrm{L}=5 \mathrm{~mm}$

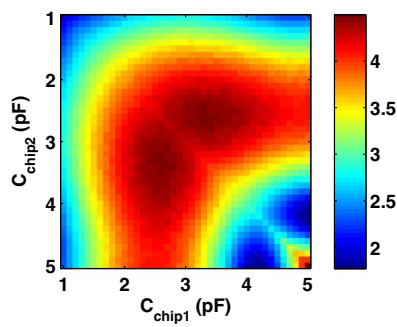

(c) $\mathrm{L}=15 \mathrm{~mm}$

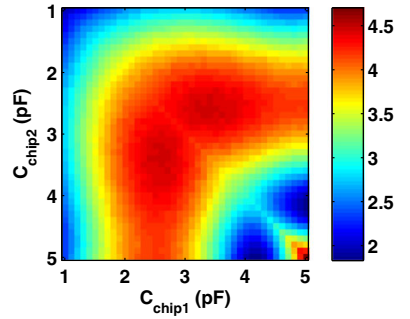

(b) $\mathrm{L}=10 \mathrm{~mm}$

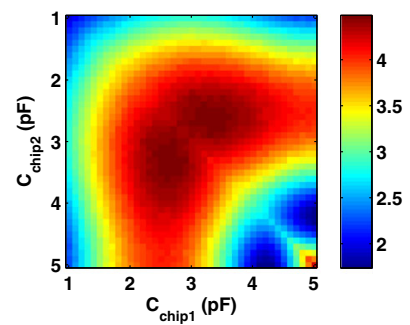

(d) $\mathrm{L}=20 \mathrm{~mm}$

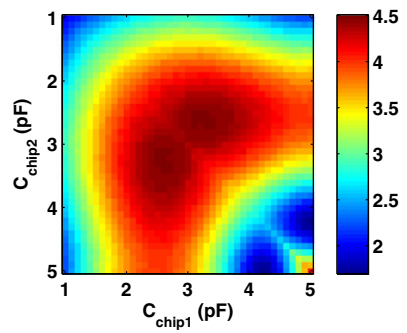

(e) $\mathrm{L}=25 \mathrm{~mm}$

Fig. 27 Standard deviation of resonance frequency $\sigma_{f_{0}}$ in (\%) when $10 \%$ standard deviation in the packaging process

Figure 30 shows the probabilities for different interconnect lengths and a $20 \%$ standard deviation in the packaging process as a function of the pair $\left(C_{\text {chip } 1}, C_{\text {chip } 2}\right)$ index, which follows the order $\{(1,1),(1,1.1),(1,1.2), \ldots$, $(4.9,5),(5,5)\}$. These probabilities are expressed in PPB (parts per billion) and indicate the high robustness of the approach due to the small values observed. The same applies to a $10 \%$ standard deviation in the packaging process, in which the probability values for a false alarm are generally null. it is also possible that the process results in $d$ deviating from its nominal value.

\subsection{Other factors impact on the resonance frequency}

The distance $d$ that separates the sensing line and the line under test is another factor that shapes the impedances $Z_{\text {even }}$ and $Z_{\text {odd }}$ and thus impacts the voltage ratio $\frac{V_{2}}{V_{g}}$. The range of the distance $d$ is generally dependent on the method of applying an isolator or a solder mask in the case of conventional PCB. Moreover,

To conclude this discussion on the relevance of this factor for the first resonance frequency of the voltage ratio, several

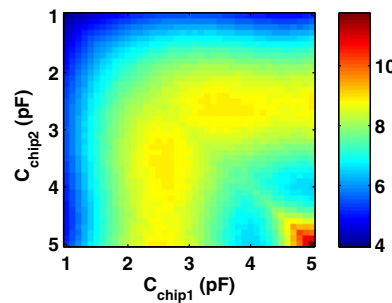

(a) $\mathrm{L}=5 \mathrm{~mm}$

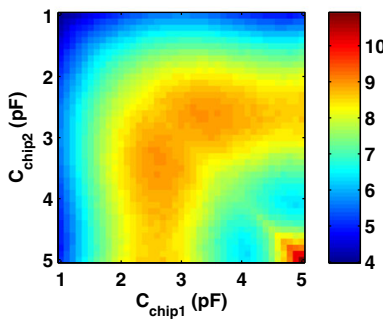

(c) $\mathrm{L}=15 \mathrm{~mm}$

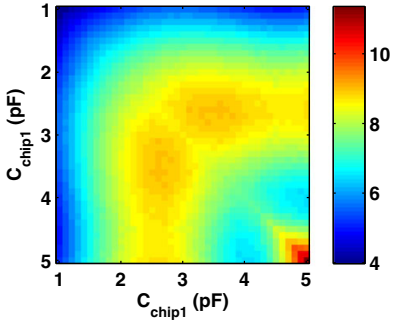

(b) $\mathrm{L}=10 \mathrm{~mm}$

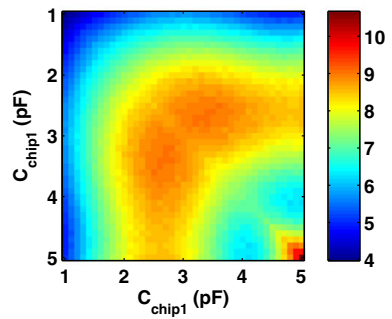

(d) $\mathrm{L}=20 \mathrm{~mm}$

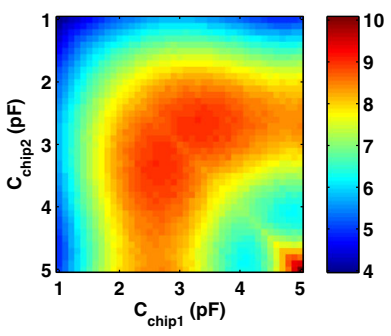

(e) $\mathrm{L}=25 \mathrm{~mm}$

Fig. 28 Standard deviation of resonance frequency $\sigma_{f_{0}}$ in (\%) when $20 \%$ standard deviation in the packaging process

cases have been verified by simulation. It has been noted that the first resonance frequency changes by negligible amounts when the distance deviates by a few micrometers from $50 \mu \mathrm{m}$ for the nominal value. In contrast, the shape

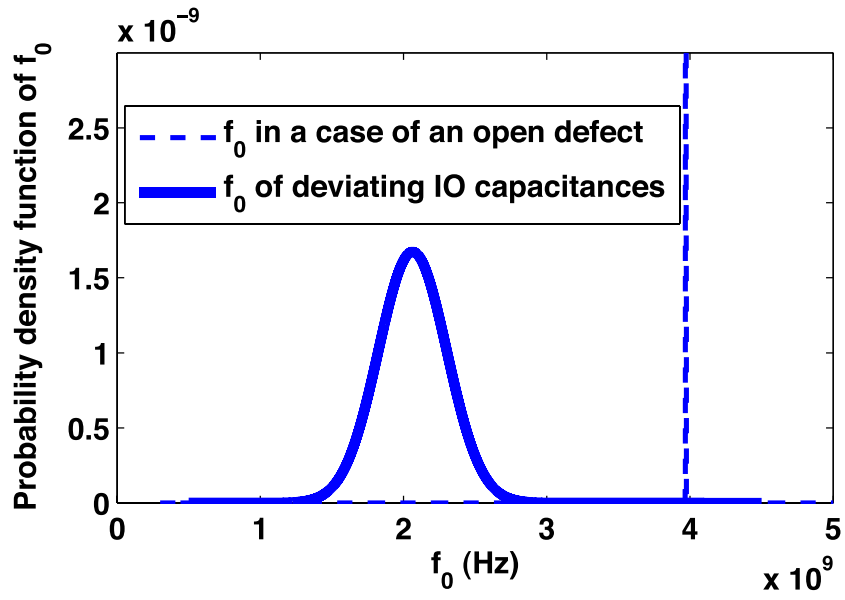

Fig. 29 Probability density function of resonance frequency $f_{0}$ 


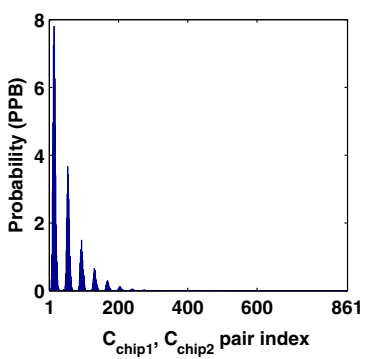

(a) $\mathrm{L}=5 \mathrm{~mm}$

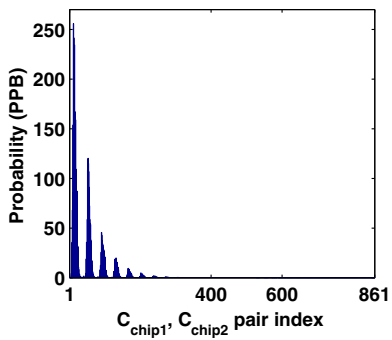

(c) $\mathrm{L}=15 \mathrm{~mm}$

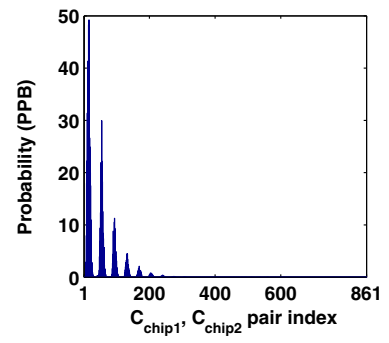

(b) $\mathrm{L}=10 \mathrm{~mm}$

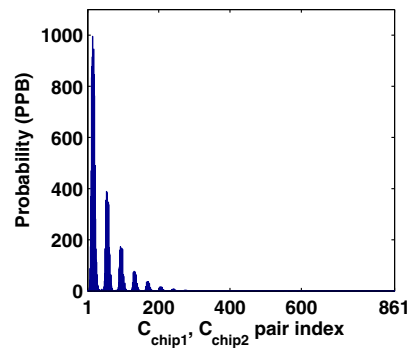

(d) $\mathrm{L}=20 \mathrm{~mm}$

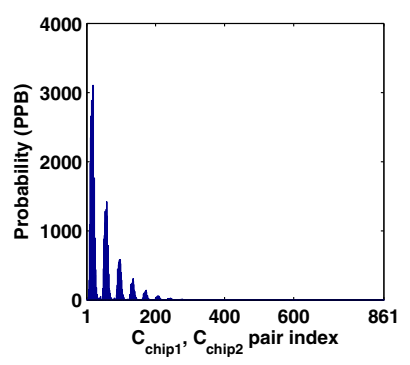

(e) $\mathrm{L}=25 \mathrm{~mm}$

Fig. 30 Probability of false alarm in PPB

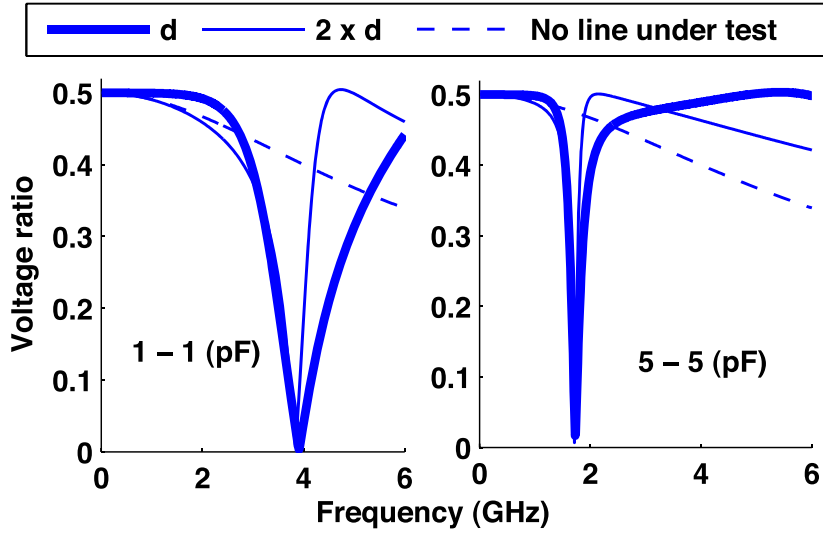

Fig. 31 Impact of the distance $d$ on the first resonance frequency in the case of 5-mm-long interconnects

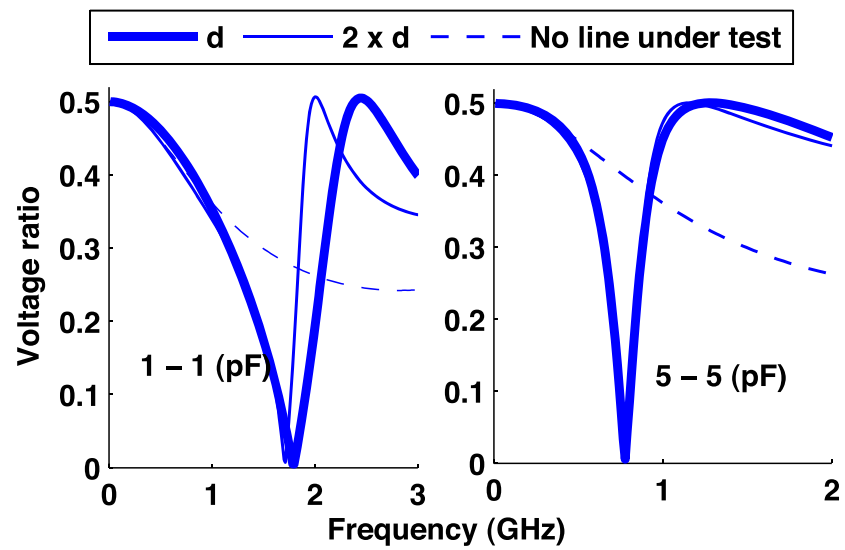

Fig. 32 Impact of the distance $d$ on the first resonance frequency in the case of 25- $\mathrm{mm}$-long interconnects

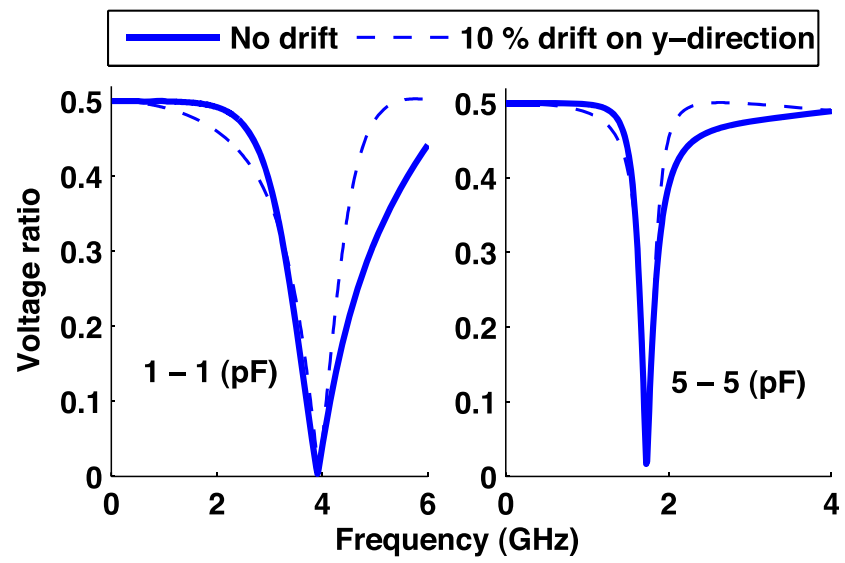

Fig. $3310 \%$ drift in y-direction of the sensing line in the case of 5$\mathrm{mm}$-long interconnects

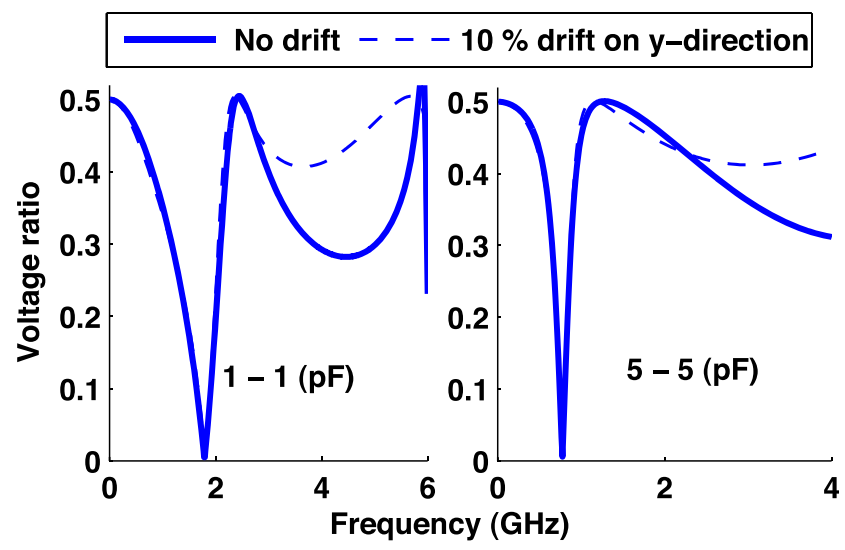

Fig. 34 10\% drift in y-direction of the sensing line in the case of 25$m m$-long interconnects 
and bandwidth of the resonating curve exhibit remarkable changes. Figures 31 and 32 demonstrate how small these variations are when the distance $d$ is double the nominal value, which is an extreme case. This indicates that the load capacitances and the coupled lengths have the most important effects on the first resonance frequency, and thus, process variations on the distance $d$ are not significant causes of false alarms. These variations might become an issue if the source of the defect discrimination is not the first resonance frequency. This can also be quickly verified by the model in [11].

Another type of factor worth verifying is the drift of the sensing line along the y-direction accounting for less than $100 \%$ of the overlapped area between the line under test and the sensing line. This can originate from the manufacturing process or from mechanical placement errors. According to Figs. 33 and 34, which have been compiled based on simulations, a $10 \%$ drift of the sensing line has no effect on the first resonance frequency for the $100 \mu \mathrm{m}$ trace width. Similar to the variations in $d$, the change in the shape of the curve is noticeable. In the practice, ground pins are provided which will serve the ground connections between the ground layers of both test bed and unit under test (UUT). They will also serve the alignment and thus the sensing lines overlap exactly with the lines under test avoiding any such parallel or non-parallel drifts.

\section{Application Sample to Conventional PCB}

Figure 35 illustrates a design which is part of a real development board that has been utilized as a UUT. This is to demonstrate the feasibility of the approach in the real use to conventional PCB. The design contains two connected BGA processors and open sockets for secure digital (SD) card and universal serial bus (USB) peripherals. The dimensions of

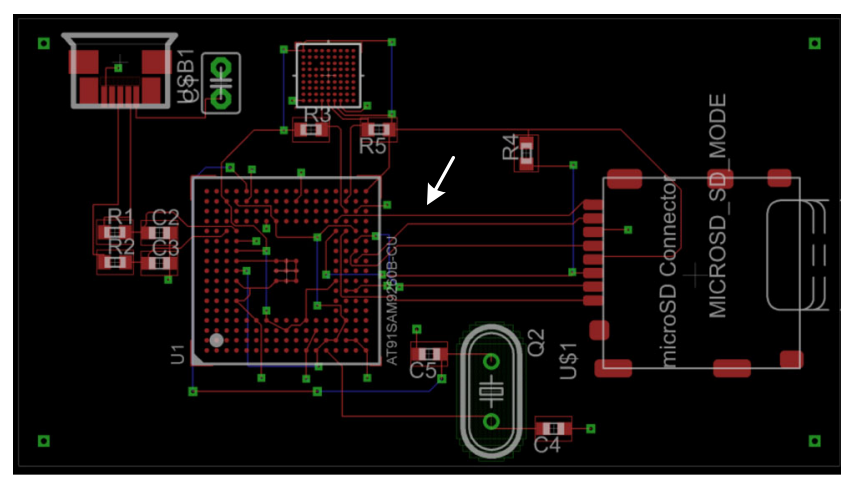

Fig. 35 Design of the UUT (a) Test bed (bottom side)

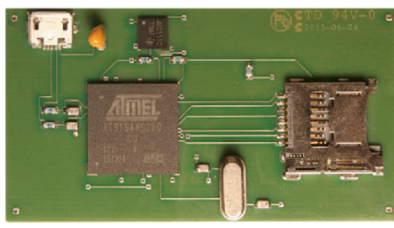

(c) UUT

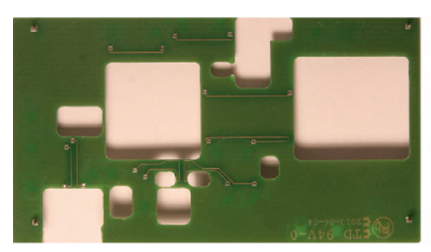

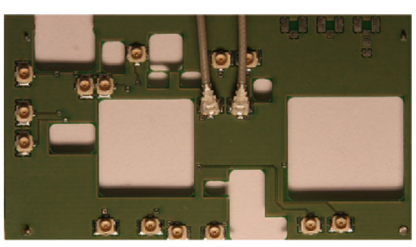

(b) Test bed (top side)

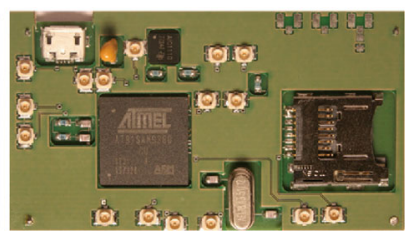

(d) Test bed mounted to the UUT
Fig. 36 Test bed and UUT

the manufactured UUT and the test bed in Fig. 36 are similar to our earlier structure description in Section 6.1, where $100 \mu \mathrm{m}$ wide and $21 \mathrm{~mm}$ long interconnect has been tested. Both UUT and test bed are double-sided boards and $511 \mu \mathrm{m}$ thick, this includes $450 \mu \mathrm{m}$ as the insulator thickness, $2 \times 18$ $\mu \mathrm{m}$ as the conducting layers thickness and about $25 \mu \mathrm{m}$ of the solder mask. The insulator is FR-4 whose permittivity is about $\varepsilon_{r}=4.3$. To ensure the broadside-coupled stripline structure, the test bed contains several cutouts which correspond to the components. Five correct and two defective boards have been assembled where opens have been introduced under the BGA package. In the defective boards, the solder pads which connect to the trace indicated by the arrow in Fig. 35 have been removed to ensure opens after the reflow process. For routing purpose and due to the nature of conventional PCB, the structure of this test case differs from the basic ones which have been simulated in such a way that there exist other parts of the line under test that cannot be made parallel to the sensing line. For this, the sensing line in the test bed covers $14 \mathrm{~mm}$ only of the line under test as shown in Fig. 37.

According to Fig. 38, the voltage ratios which have been taken using the network analyzer follow the concept of the first resonance frequency, an open decreases the load

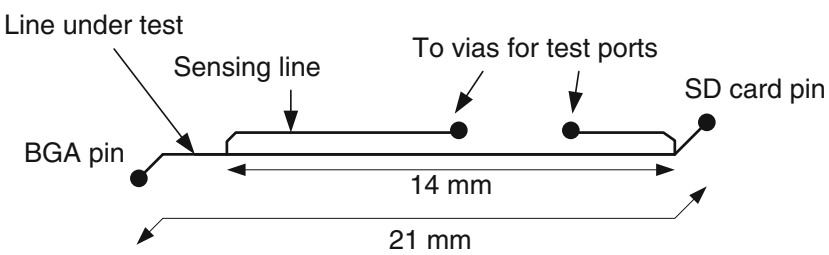

Fig. 37 Test case structure 


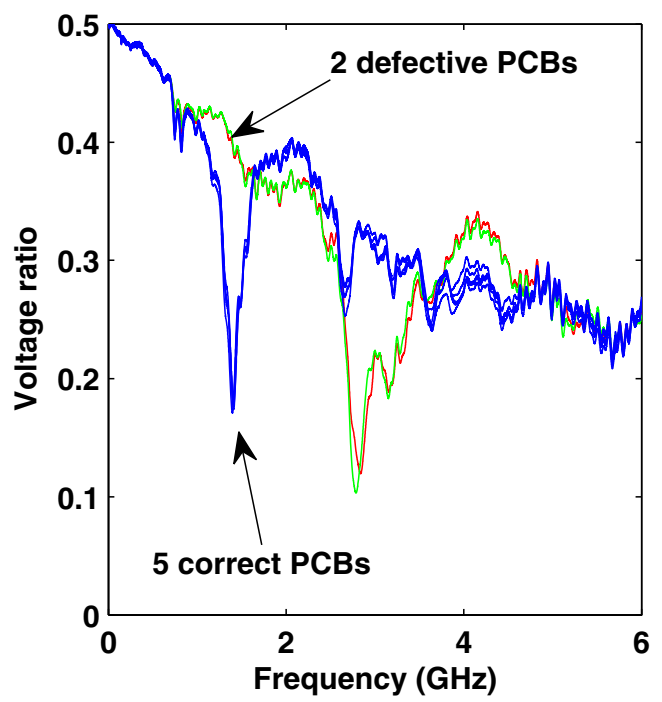

Fig. 38 Test bed and a UUT have been assembled

capacitance and thus an increase in the first resonance frequency where the voltage ratio goes towards zero. Although other oscillations are occurring due to the differences in the structure and possible cross talk between the interconnect under test and the other solder balls. Anyhow, a significant margin can be achieved around the resonance frequency which is the potential source of discriminating the defect.

\section{Realistic Structure for Conventional PCBs}

When applying the contactless approach to conventional PCBs, we do not end up with only two coupled line structures in most cases. The transmission lines that allow us to connect to the sensing line and the component pins exist, e.g., the solder balls. Specifically, those that do not exist at the edge of the package always involve extension lines, as illustrated in Fig. 39 by the orange color, and those extension lines are referred to as $T L_{3}$ and $T L_{4}$ for the traces of the UUT.

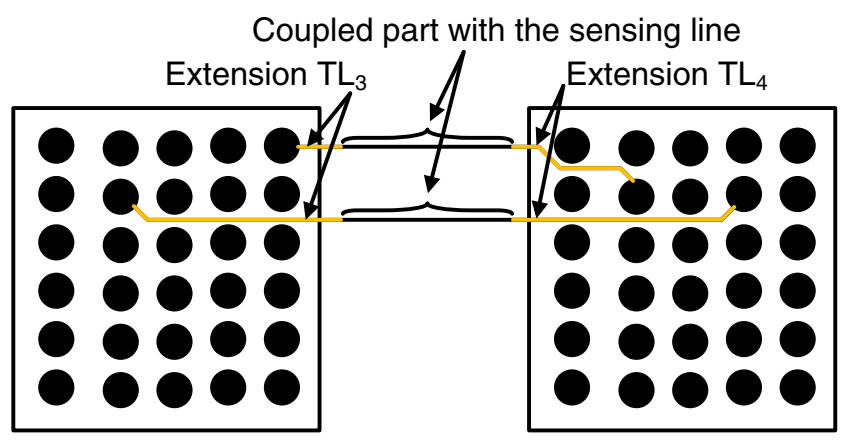

Fig. 39 Samples of two BGA interconnects in the tests with extensions

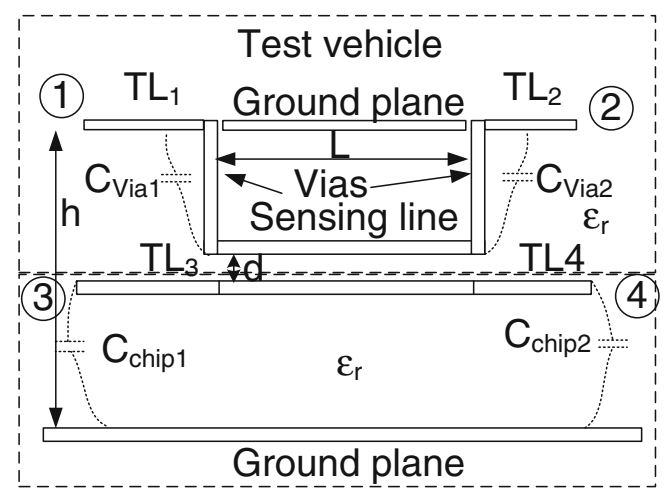

Fig. 40 Voltage ratio between ports 2 and 1 for trace in question within seven PCBs

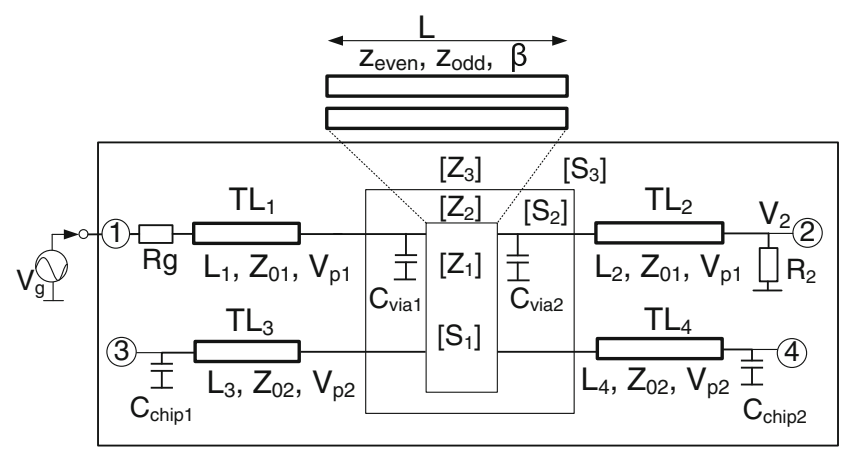

Fig. 41 Realistic model of the test vehicle and UUT with vias and extensions

In this section, an improved model is developed that considers these transmission lines connecting port 1 to the generator and port 2 to the measurement device as well as the extensions connecting the component pins to the coupled part. Figure 40 shows the structure of the complete model, while Fig. 41 shows the electrical model with the extension transmission lines of the sensing line $T L_{1}$ and $T L_{2}$, whose lengths, characteristic impedance and propagation speed are $L_{1}, L_{2}, Z_{01}$ and $V_{p 1}$, respectively. In addition, the extension transmission lines of the line under test are $T L_{3}$ and $T L_{4}$, whose lengths, characteristic impedance and propagation speed are $L_{3}, L_{4}, Z_{02}$ and $V_{p 2}$, respectively.

\section{PEEC Simulations}

According to the results in Section 6, a significant margin is obtained between the resonance frequency of a defect-free termination and an open termination, which is represented by $0.5 \mathrm{pF}$. As an assumption for the current work, as long as the extensions are negligible, the results of these resonance frequencies of the voltage ratio should agree with the results obtained in Section 6. 
To proceed with the effect of the extensions $T L_{1}, T L_{2}$, $T L_{3}$ and $T L_{4}$ on the previous results, we have modeled and simulated a number of variations to this using a multi-PEEC EM solver [24]. The varied parameters are as follows:

- Length of interconnect: 5 - $25 \mathrm{~mm}$ with a 5-mm step.

- Load capacitance: any value from the set $\left\{\begin{array}{llll}0.5 & 1 & 1.5 & \ldots\end{array}\right.$ $4.55\} \mathrm{pF}$.

- The extensions $L_{3}$ and $L_{4}$ can be any pair from the set of values $\left\{\begin{array}{lllllll}0.1 & 1 & 2 & 3 \ldots 13 & 14 & 25\end{array}\right\} \mathrm{mm}$, which makes 676 pairs in total. A $25-\mathrm{mm}$ extension could be the maximum extension under the package or inside an inner layer for today's electronics.

- Extensions $L_{1}$ and $L_{2}$ have been selected as one of the following pairs: $(0.1,0.1),(5,5)$ and $(15,15) \mathrm{mm}$.

The main reason for choosing only three pairs for $L_{1}$ and $L_{2}$ is that it is expected that the first resonance frequency of the voltage ratio will remain constant as has been noted, and it was independent of $C_{V i a 1}$ and $C_{V i a 2}$.

During the simulation, the partial inductances $L_{p}$, the coefficients of the potential $P$ and the delay $\tau$ have been considered as PEEC simulation parameters [1]. For 5- to 25- $m m$-long broadside-coupled striplines, the partial resistances $R$ are too small for the structure and are worth neglecting for our purpose. Thus, a proper full-wave modeling of the problems has been simulated.

The simulated structure, which is shown in Fig. 40, reflects today's industry capabilities for miniaturization, where the trace width is $100 \mu \mathrm{m}$ and the PWB thickness is $450 \mu \mathrm{m}$, which similarly applies to the board of the sensing line. The gap between the sensing line and the interconnect under test is represented as $50 \mu \mathrm{m}$, which corresponds roughly to both solder mask thicknesses.

To determine the voltage ratio for the whole system, we use:

$\frac{V_{2}}{V_{g}}=\frac{z 3_{21}}{z 3_{11}}$

A method described in [26] was employed to determine the scattering parameters $\left[S_{3}\right]$ for the whole system and the impedance matrix $\left[Z_{3}\right]$.Let $C_{3}$ and $C_{4}$ denote the chip capacitances $C_{c h i p 1}$ and $C_{c h i p 2}$, respectively.

\subsection{Impact of extensions at the UUT side}

Figures 42, 43, 44, 45 and 46 show the first resonance frequency for different coupled lengths and different extensions at the UUT. It is clearly shown that the highest

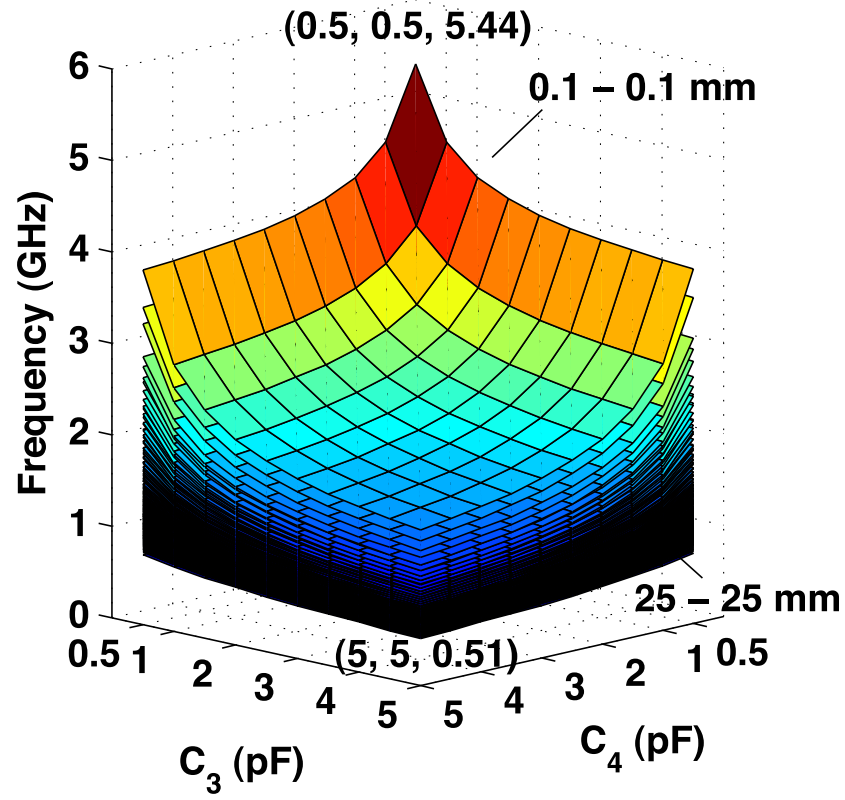

Fig. $42[\mathrm{Z}]$ and $[\mathrm{S}]$ parameters for the complete electrical model

extensions at the UUT lead to the lowest first resonance frequency. In contrast, the lowest extensions lead to the highest first resonance frequency.

To enable a quick snapshot of the needed frequency range for a specific coupled length and extensions in the UUT, Fig. 47 can be utilized, where the extensions in the UUT are quantified by Ratio $=\frac{L_{3}+L_{4}}{L}$.

For a given length $L$ and Ratio, a frequency range that covers $f 0_{\max }$ is needed to test for capacitive terminations in

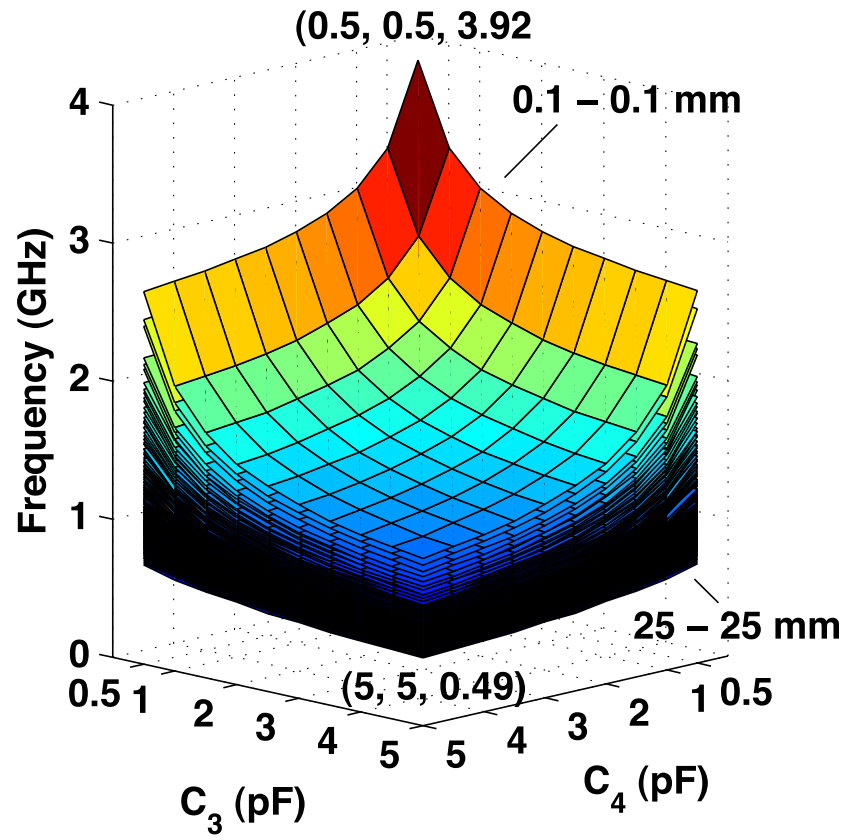

Fig. 43 First resonance frequency versus $C_{3}$ and $C_{4}$ for $5 \mathrm{~mm}$ and different extension combinations of $T L_{3}$ and $T L_{4}$ 


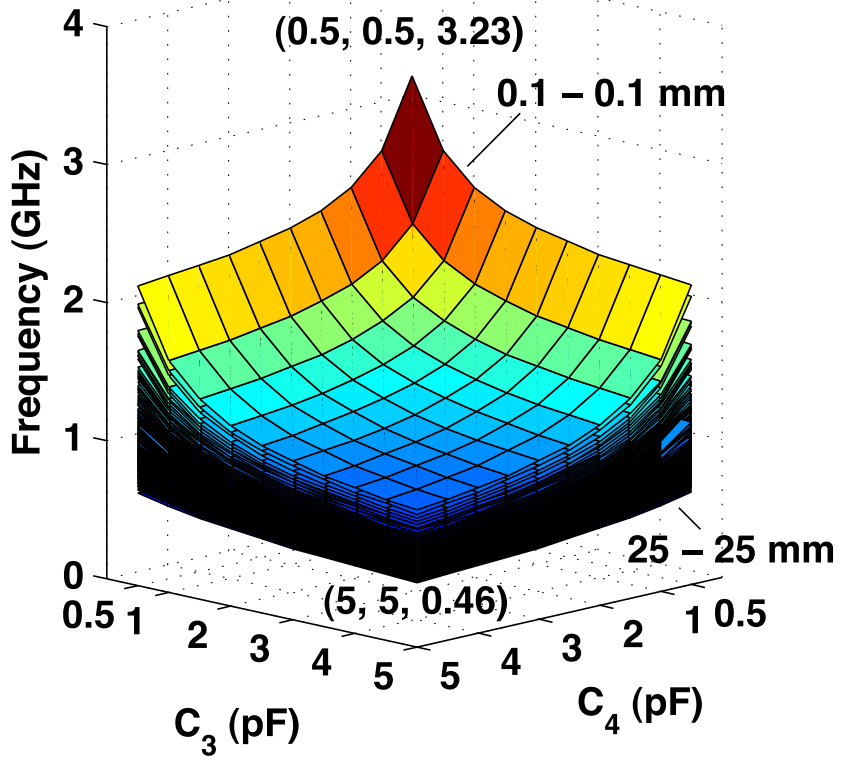

Fig. 44 First resonance frequency versus $C_{3}$ and $C_{4}$ for $10 \mathrm{~mm}$ and different extension combinations of $T L_{3}$ and $T L_{4}$

the range of $[0.5 \mathrm{pF}, 5 \mathrm{pF}] . f 0_{\max }$ corresponds to the case in which no extensions at the UUT and both terminations are represented by $0.5 \mathrm{pF}$. By increasing the extensions, the boundaries of the surface $\left(f 0_{\max }\right.$ and $\left.f 0_{\min }\right)$ decrease. According to the simulation results, the extensions at the UUT play a positive role. By increasing the extensions,

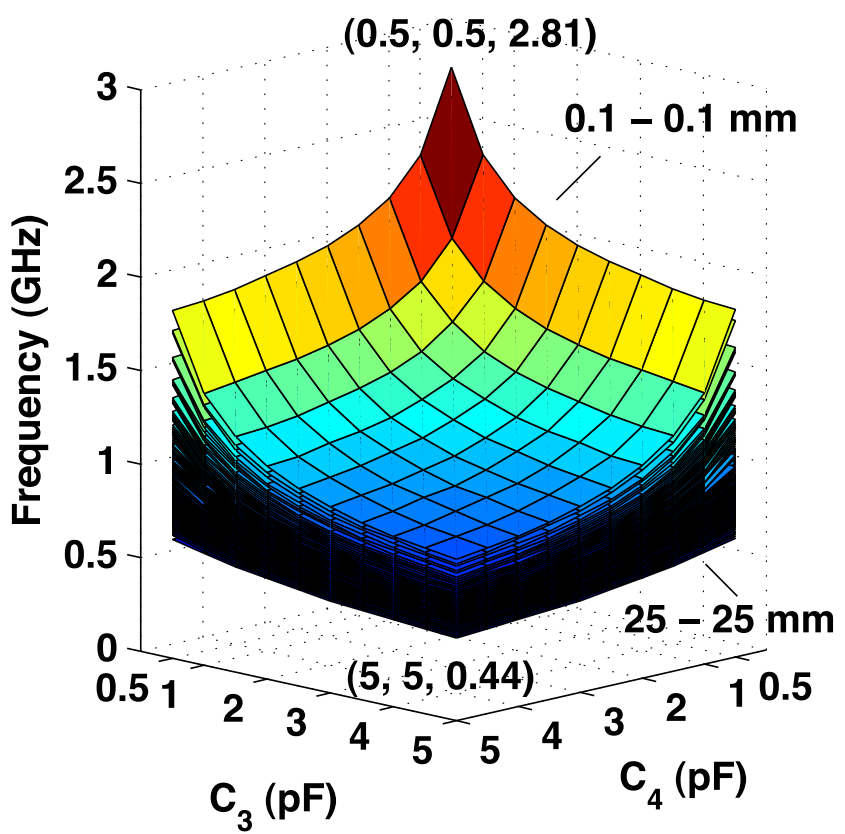

Fig. 45 First resonance frequency versus $C_{3}$ and $C_{4}$ for $15 \mathrm{~mm}$ and different extension combinations of $T L_{3}$ and $T L_{4}$

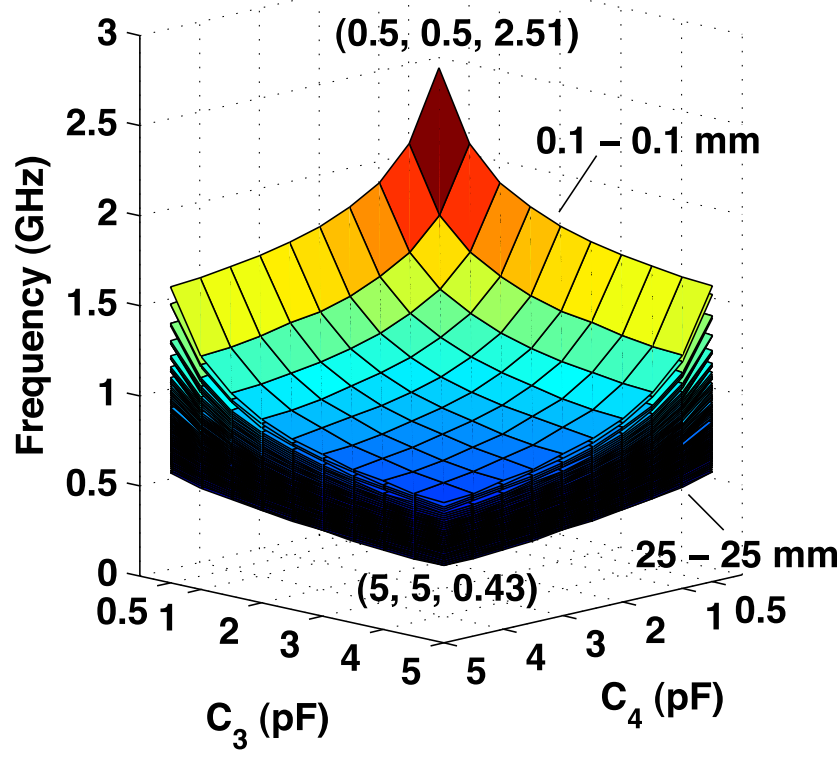

Fig. 46 First resonance frequency versus $C_{3}$ and $C_{4}$ for $20 \mathrm{~mm}$ and different extension combinations of $T L_{3}$ and $T L_{4}$

the first resonance frequency is reduced, which might be preferable in some cases.

\subsection{Impact of extensions at the sensing line side}

Only equal lengths of $T L_{1}$ and $T L_{2}$ were used as pairs during the simulations. For $L_{1}=L_{2}=0.1 \mathrm{~mm}, L_{1}=L_{2}=$ $5 \mathrm{~mm}$ and $L_{1}=L_{2}=15 \mathrm{~mm}$, the simulation results are completely identical. The extensions at the sensing line have no impact on the first resonance frequency. This phenomenon is hard to be proven true analytically using the theoretical model of two coupled lines which has been reported in [11]. To derive the voltage ratio $\frac{V_{2}}{V_{g}}$ analytically, cascading the extensions with the theoretical model and the required terminations is a key, however the procedure is heavy as it involves $4 \times 4$ matrix inversions with many variables which makes it unfeasible and thus numerical

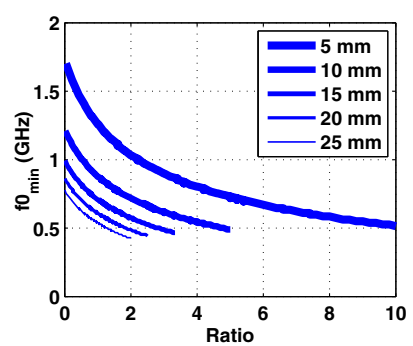

(a)

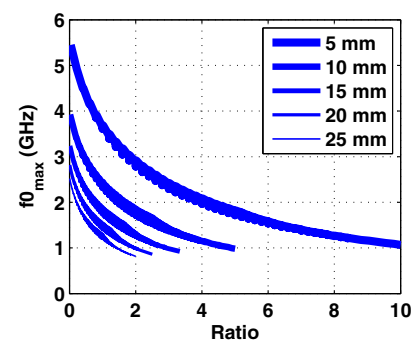

(b)
Fig. 47 Minimum and maximum first resonance frequency as function of the ratio for 5 to $25 \mathrm{~mm}$ coupled lengths 
solutions, simulations and measurements remain the only options.

\subsection{Measurement samples}

To verify the impact of the extensions experimentally, several samples which include a UUT and a sensing line of 5 and $10 \mathrm{~mm}$ as coupled lengths were prototyped. The structure model follows the Fig. 40 in Section 11. The sample PCBs feature two broadside striplines, which are connected to the top and bottom layers through $300 \mu \mathrm{m}$ wide vias. The ground planes are separated by a distance $h=0.986 \mathrm{~mm}$, which includes the gap between the coupled lines, $d=50 \mu \mathrm{m}$; the gaps between the lines and the ground planes, $2 \times 0.45 \mathrm{~mm}$; and the thickness of the copper layer, $2 \times 18 \mu \mathrm{m}$, where FR-4 with $\varepsilon_{r}=4.3$ is used. To cover a possible extensions range, the measurements involve six cases of each coupled length.

As the purpose of these measurements is to confirm with few examples the impact of the extensions on the first resonance frequency, the line under test was all the time terminated by $5.6 \mathrm{pF}$ as $C_{3}$ and $C_{4}$ in addition to the capacitance of the via. Figures 48 and 49 confirm the simulation remarks on the impact of the extensions at the sensing line side on the first resonance frequency. Within all these cases, the first resonance frequency is independent of the upper extensions $L_{1}$ and $L_{2}$. On the other hand, increasing the ratio $\frac{L_{3}+L_{4}}{L}$ decreases the first resonance frequency of the voltage ratio as concluded by simulations.
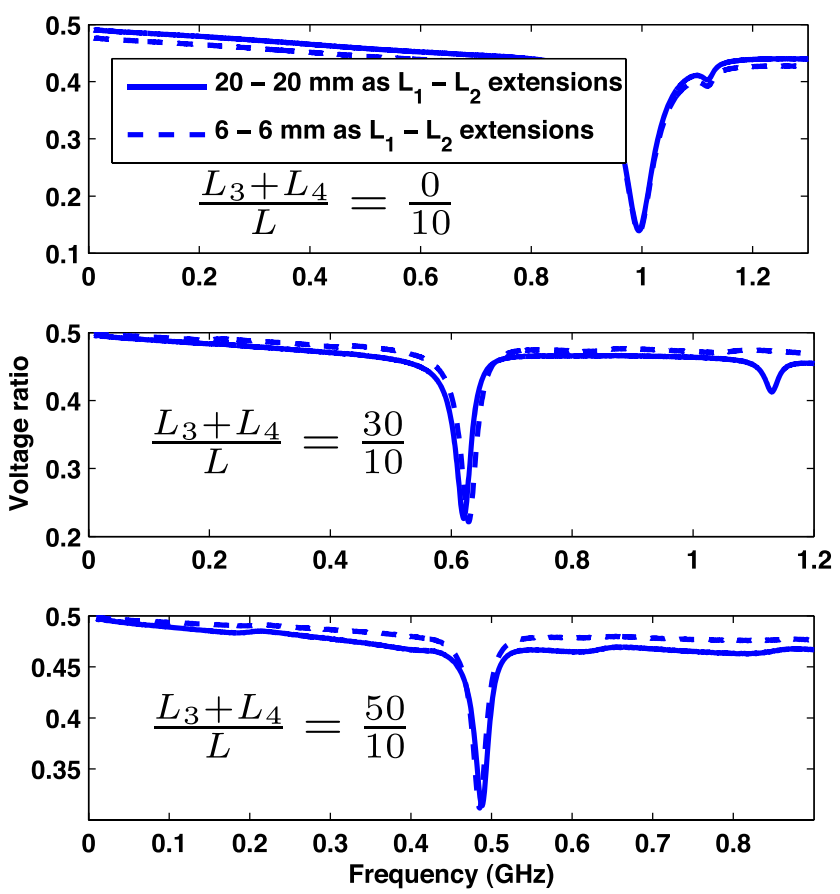

Fig. 48 First resonance frequency versus $C_{3}$ and $C_{4}$ for $5 \mathrm{~mm}$ and different extension combinations of $T L_{3}$ and $T L_{4}$
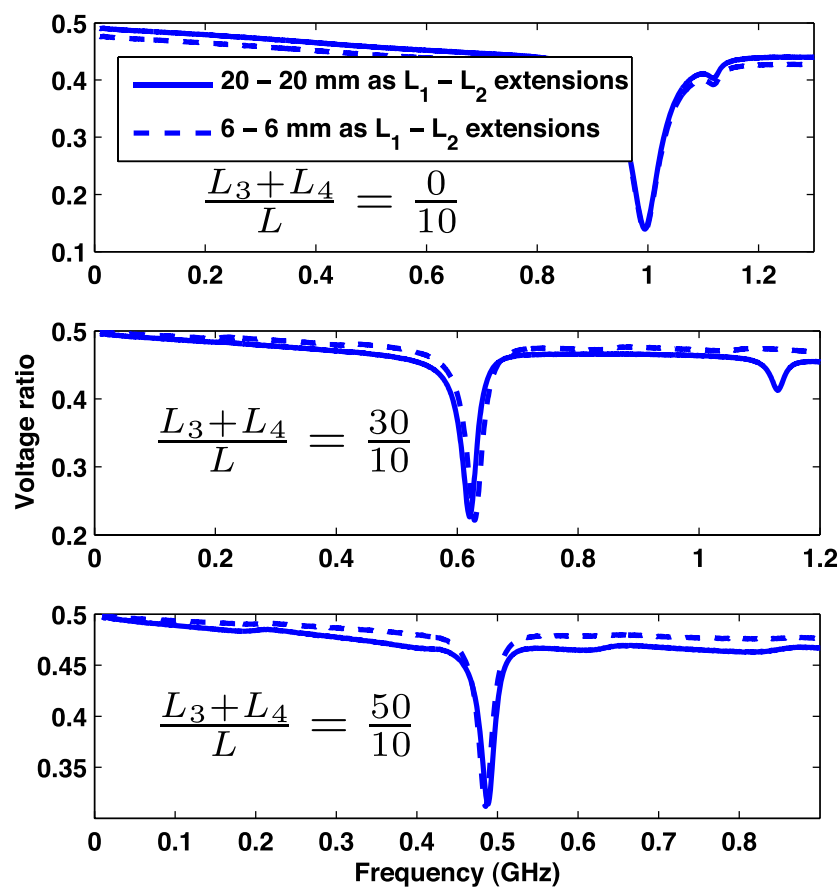

Fig. 49 First resonance frequency of the voltage ratio for $5 \mathrm{~mm}$ coupled length and different extensionsFirst resonance frequency of the voltage ratio for $10 \mathrm{~mm}$ coupled length and different extensions

\section{Design for Testability (DfT) Rules}

According to the first resonance frequency Figs. (42, 43, 44,45 and 46) of the different coupled lengths and extensions, the margin always exists between the first resonance frequencies of the defective and error-free interconnects.

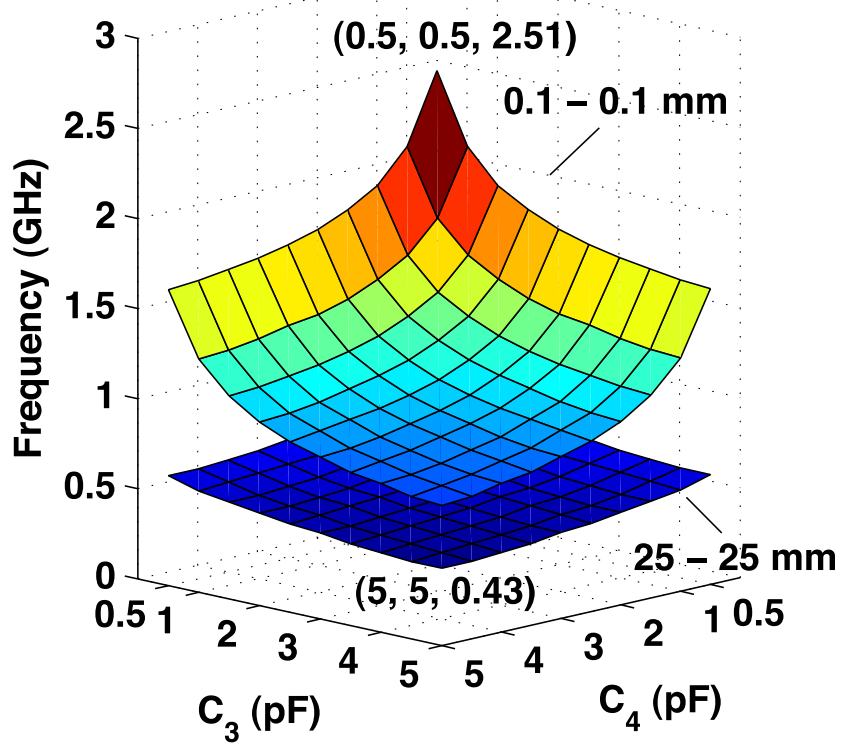

Fig. 50 A higher ratio $\frac{L_{3}+L_{4}}{L}$ leads to a flatter surface and thus lower frequency margins 
It is clear that the margin between the first resonance frequency, which corresponds to an error-free interconnect, and that of an erroneous interconnect increases when the extensions or the ratio $\frac{L_{3}+L_{4}}{L}$ decrease. A higher ratio results in a flatter obtained surface and, thus, a smaller margin, as illustrated in Fig. 50. However, the point of interest is the approach ability in detecting open defects with manageable margins. In this section, DfT rules are derived to be considered during the design phase for robust open defect detection.

\subsection{DfT for $10 \%$ margin}

In this section, a model that determines the maximum ratio has been created with $10 \%$ as the minimum measurable margin. Figure 51a, b, c, d and e illustrate the scattered minimum margins that can be obtained for all possible ratios for each coupled length. These ratios represent any combination pair of the extensions $L_{3}$ and $L_{4}$, where $0.1 \mathrm{~mm}$ is the minimum and $25 \mathrm{~mm}$ is the maximum with $1-\mathrm{mm}$ steps. It is suspected that most of the minimum margins occur at the index of $\left(C_{3}, C_{4}\right)$, which corresponds to $(1,1) \mathrm{pF}$. The rectangle in each figure dictates the maximum possible ratio that allows for the minimum margin of $10 \%$.

Figure 52 presents a model that can be applied during DfT using the rule of $10 \%$ as the minimum margin in the first resonance frequency. The model Ratio $_{\max }=45.75 \mathrm{~L}^{-1.4704}$ is in good agreement with the simulation data, where $L$ is in $\mathrm{mm}$.

\subsection{DfT for packaging error}

Manufacturing process deviations lead to IO capacitance deviations and thus a shift in the first resonance frequency. According to Fig. 27, a 10\% standard deviation in the IO capacitance leads to a standard deviation $\sigma$ in the first resonance frequency, whereas it is $2 \% \leq \sigma \leq 5 \%$ on average, as shown in Fig. 27. Under the assumption that these deviations do not increase with the extensions, in this section, a model that determines the maximum ratio has been created to tolerate $3 \times \sigma$ as the minimum measurable margin. This tolerance is needed to ensure that the measurement device do not fail a correct trace because of the manufacturing process deviations in the package. Figure 53a, b, c, $\mathrm{d}$ and e illustrate the scattered minimum margins that can be obtained for all possible ratios for each coupled length. These ratios represent any combination pairs of the extensions $L_{3}$ and $L_{4}$, where $0.1 \mathrm{~mm}$ is the minimum and $25 \mathrm{~mm}$ is the maximum with a $1 \mathrm{~mm}$ step. It is suspected that

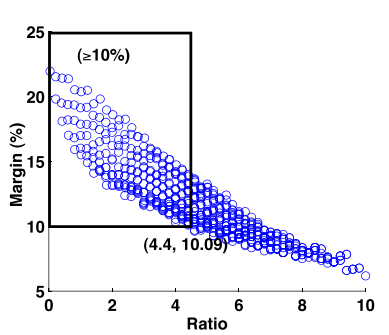

(a) $\mathrm{L}=5 \mathrm{~mm}$

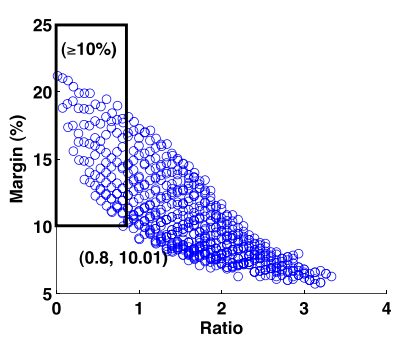

(c) $\mathrm{L}=15 \mathrm{~mm}$

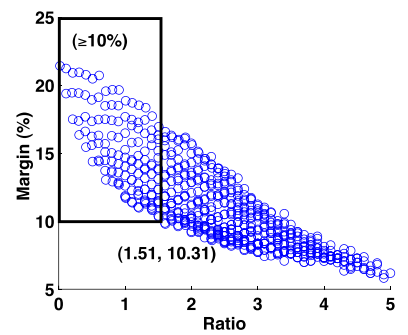

(b) $\mathrm{L}=10 \mathrm{~mm}$

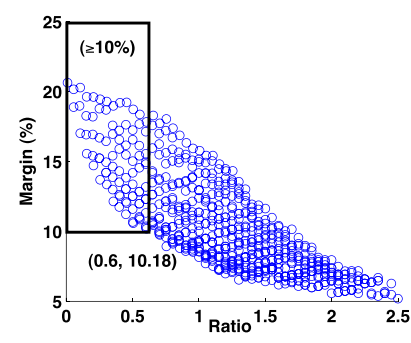

(d) $\mathrm{L}=20 \mathrm{~mm}$

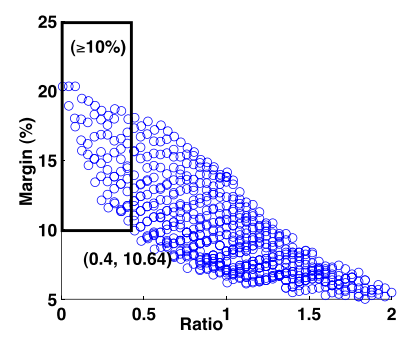

(e) $\mathrm{L}=25 \mathrm{~mm}$

Fig. 51 Minimum margin that can be obtained for all possible ratios

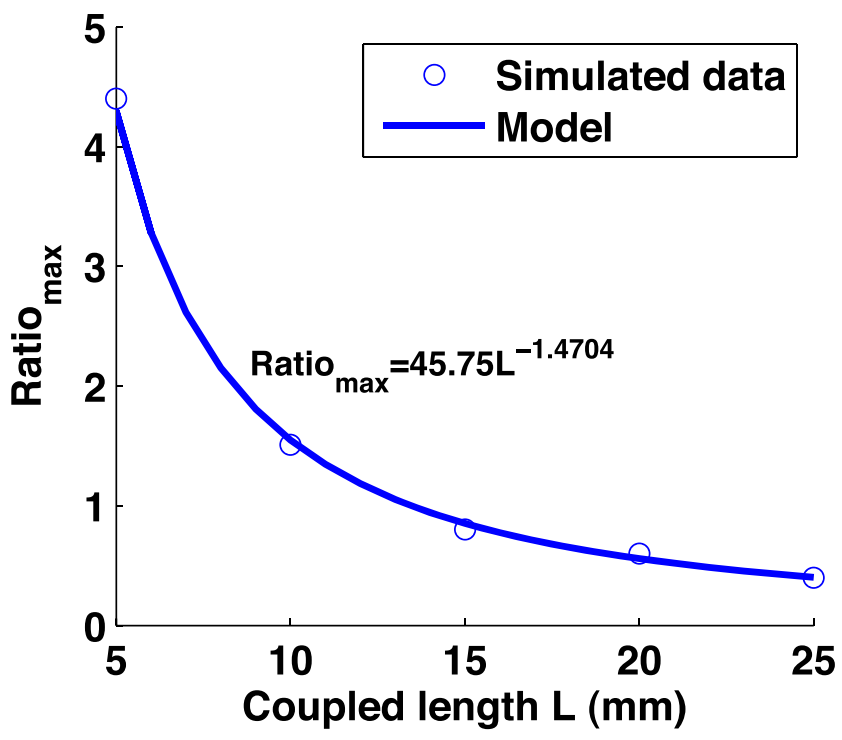

Fig. 52 DfT rule model for a 10\% margin 


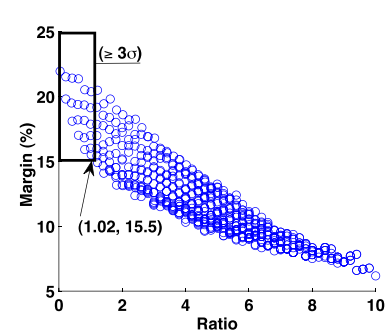

(a) $\mathrm{L}=5 \mathrm{~mm}$

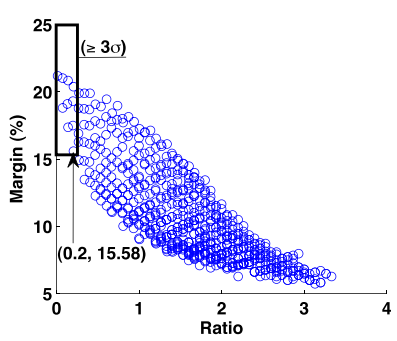

(c) $\mathrm{L}=15 \mathrm{~mm}$

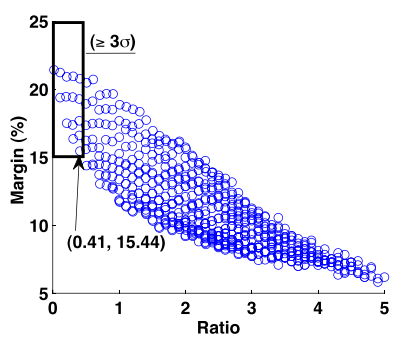

(b) $\mathrm{L}=10 \mathrm{~mm}$

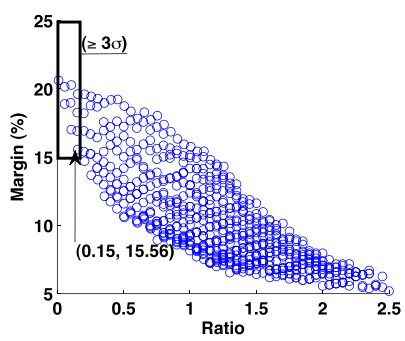

(d) $\mathrm{L}=20 \mathrm{~mm}$

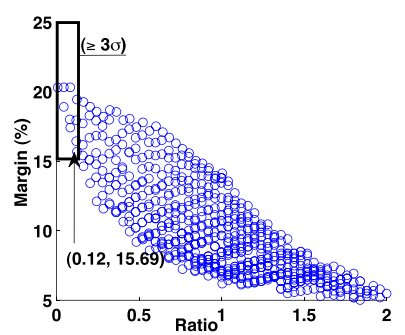

(e) $\mathrm{L}=25 \mathrm{~mm}$

Fig. 53 Minimum margin that can be obtained for all possible ratios

most of the minimum margins occur at the index of $\left(C_{3}\right.$, $\left.C_{4}\right)$, which corresponds to $(1,1) \mathrm{pF}$. The rectangle in each figure determines the maximum possible ratio that allows a minimum margin of $3 \times \sigma$. Figure 54 presents a model that can be applied during DfT as a rule for $3 \times \sigma$ as

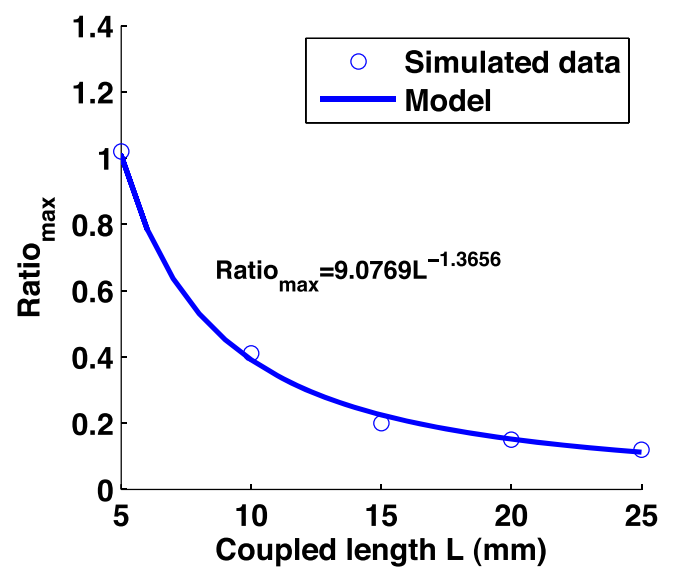

Fig. 54 DfT rule model for $3 \times \sigma$ the minimum margin in the first resonance frequency. The model Ratio $_{\max }=9.0769 L^{-1.3656}$ is in good agreement with the simulation data, where $L$ is in $\mathrm{mm}$.

\section{Test Performance and Limitations}

Let the intermediate frequency bandwidth (IFBW) of a network analyzer be $100 \mathrm{KHz}$. For the maximum measurement frequency of $6 \mathrm{GHz}$ and for a sampling step of $600 \mathrm{KHz}$ which is fine enough, the number of sampling points is $\frac{6 \mathrm{GHz}}{600 \mathrm{KHz}}=10,000$ which are distributed linearly over the bandwidth. The test time for one interconnect will be approximately

points $\times \frac{1}{I F B W}=100 \mathrm{~ms} \Leftrightarrow 10 \mathrm{~s} / 100$ interconnects

The switching hardware will also add some overhead to the computed test time. The switching time of an electromechanical switch is expressed in milliseconds and this latter is much slower as compared to a solid state one whose switching time is expressed in nanoseconds. If we assume that the electromechanical switches are employed which are in favor in terms of their insertion loss and isolation as compared to the solid state ones [10]. The switching architecture can be made by cascading the basic RF single pole changeover (SPCO) switch. Generally, the switching time of the mainstream of this type of RF switches is much less than $20 \mathrm{~ms}$. Taking $20 \mathrm{~ms}$ as the worst case scenario, the switching will cost 2 s100, interconnects as the switches are controlled at the same time. This makes the total test time to be $12 \mathrm{~s} / 100$ interconnects , thus the switching time is not dominant even when employing the slowest electromechanical switches.

This time can be reduced significantly using prior knowledge of the first resonance frequency, which reduces the test frequency and the number of samples [3]. Obviously, there will be other overheads, but the one that will dominate the test performance is the time taken to automatically place the test bed on the top of the board under test. Another way to accelerate testing is deploying, for this purpose, several application-specific network analyzers and using different IFBWs to avoid interference between the measurements.

Due to the high density of the interconnects, one might not be able to place all the RF connectors that connect the sensing lines to the network analyzer. To overcome this challenge, for a board under test, several different test beds might need to be designed, and each will consist of a set of sensing lines that are sufficiently far from each other 
to allow space for miniature RF connectors and to ensure crosstalk avoidance. Otherwise, high number of interconnects affect only the test time when the traces are distributed at low density on the board. In such cases, only one or a reasonable number of test beds are needed. For multilayer boards, inner layer interconnects must be brought partially to the upper layer for testing, which is one fundamental limitation of this approach.

For this study, only open defects were analyzed. This is because assembling electronics involves creating connections between device pins and interconnects. We may not consider bridge defects if the process initially fails to connect components together. However, a bridge defect will increase the load capacitance, which will decrease the first resonance frequency of the voltage ratio $\frac{V_{2}}{V_{g}}$. This does not limit the feasibility of this approach to only detecting open defects.

\section{Conclusion}

A new approach to the testing of high density interconnect is proposed. By simulation and by direct measurements, it has been shown that the approach is feasible for examples reflecting current state-of-the-art HDI. The method provides robust error detection margins allowing for detecting faults in the range of a few PPB while accounting for component specification variabilities of $10-20 \%$. By simulations, it has also been shown that the method is robust against reasonable variations in the $\mathrm{z}$ - and $\mathrm{y}$-direction, which translate into variations in the separating insulator thickness and into the mechanical placement error of the sensing line. To apply this approach, the maximum test frequency is around $6 \mathrm{GHz}$, which is manageable. In situations where the current test solutions are not feasible, the introduced approach has a great potential for better defect coverage.

The impact of interconnect extensions has been studied for a test structure that is believed to be the most common in today's PCBs. Extensions reduce the first resonance frequency and decrease the margin between the resonance frequencies of correct and defective interconnects. Within 5- to 25-mm-long interconnects and with between 0.1 and $25 \mathrm{~mm}$ extensions at UUT, DfT rules for the extensions are derived that tolerate $10 \%$ packaging deviations and $10 \%$ as the minimum possible margin for defect detection.

\section{References}

1. Antonini G, Delsing J, Ekman J, Orlandi A, Ruehli A (2007) PEEC development road map 2007. Tech Rep 5. Lulea University of Technology
2. Davis B (1994) Economic modeling of board test strategies. J Electron Test 5(2-3):157-169

3. Dunsmore JP (2012) Handbook of microwave component measurements: with advanced VNA techniques, 1st. Wiley, chap 2-5

4. Fjelstad J (2009) An alternative process for assembling electronic products without solder. Advancing Microelectronics 36(4):18-21

5. Fjelstad J (2009) Solderless assembly of electronic products - a more reliable and more cost effective approach to electronics manufacturing? In: IEEE Vehicle power and propulsion conference, 2009 VPPC,09. IEEE, pp 11-16

6. Fjelstad J (2011) Benefits of reversing the circuit manufacturing and assembly processes for electronic products. In: IEEE international symposium on Electromagnetic compatibility (EMC), 2011. IEEE, pp 624-628

7. Hall SH, Heck HL (2009) Advanced signal integrity for highspeed digital designs. Network analysis for digital engineers, 1st edn. Wiley, pp 347-415

8. Hannu J, Häkkinen J, Voutilainen JV, Jantunen H, Moilanen M (2012) Current state of the mixed-signal test bus 1149.4. J Electron Test 28(6):857-863

9. Holden H (2009) The HDI Handbook. Embedded Components, 1st edn. BR Publishing, Inc., pp 504-506

10. Johler W (2003) RF performance of ultra-miniature high frequency relays. In: Proceedings of the forty-ninth IEEE holm conference on electrical contacts, 2003. pp 179-189

11. Jones E (1956) Coupled-strip-transmission-line filters and directional couplers. IRE Transactions on Microwave Theory and Techniques 4(2):75-81

12. Kim B, Choi HH (2001) A new test method for embedded passives in high density package substrates. In: Proceedings of the 51st IEEE electronic components and technology conference, 2001. pp 1362-1366

13. Kim GS, Takamiya M, Sakurai T (2009) A capacitive coupling interface with high sensitivity for wireless wafer testing. In: IEEE international conference on 3D system integration, 3DIC, 2009. pp $1-5$

14. Kim J, Kim H, Kim S, Bae B, Jung D, Kong S, Kim J, Lee J, Park K (2013) Non-contact wafer-level tsv connectivity test methodology using magnetic coupling. In: IEEE international 3D systems integration conference, (3DIC), 2013. pp 1-4

15. Kujala A, Tuominen R, Kivilahti J (1999) Solderless interconnection and packaging technique for embedded active components. In: Proceedings of the 49th IEEE electronic components and technology conference, 1999. pp $155-159$

16. Larsson K, Pettersson A, Hedlund S, Forsling W, Gunneriusson L, Lindberg M (2001) Method of applying metal layers in distinct patterns. US Patent 6,303,278. https://www.google.com/patents/ US6303278

17. Ley A (2012) Solving the problem of diminishing test coverage from in-circuit test (ICT). Tech. Rep. 17, ASSET InterTech

18. Marinissen E, Lee DY, Hayes J, Sellathamby C, Moore B, Slupsky S, Pujol L (2009) Contactless testing: possibility or pipe-dream? In: Design, automation test in europe conference exhibition, 2009. Date '09., IEEE, pp 676-681

19. Moore B, Sellathamby C, Cauvet P, Fleury H, Paulson M, Reja M, Fu L, Bai B, Reid E, Filanovsky I, Slupsky S (2007) High throughput non-contact $\mathrm{SiP}$ testing. In: IEEE international test conference, ITC, 2007. pp 1-10

20. Noguchi M, Tsunoda O, Tomita H, Saito Y (2012) Study for a defect detection system with capacitance on the printed wiring board. In: IEEE 13th internatinal workshop on mechatronics (MECATRONICS), 9th France-Japan 7th Europe-Asia Congress 
on and research and education in mechatronics (REM), 2012. pp 182-187

21. Norrgard D, Parker KP (2008) Augmenting boundary-scan tests for enhanced defect coverage. In: IEEE international test conference, ITC, 2008. pp 1-8

22. Parker KP (2003) The Boundary-Scan Handbook, 3rd edn. Springer US, chap IEEE 1149.4: Analog Boundary-Scan

23. Parker KP (2012) Capacitive sensing testability in complex memory devices. In: IEEE international test conference (ITC), 2012. pp 1-6

24. Ruehli A, Antonini G, Esch J, Ekman J, Mayo A, Orlandi A (2003) Nonorthogonal PEEC formulation for time- and frequencydomain EM and circuit modeling. IEEE Trans Electromagn Compat 45(2):167-176

25. Scheiber SF (2001) Building a successful board-test strategy, Newnes, chap What is a test strategy?, 2nd edn., pp 1-4

26. Simpson G (1981) A generalized n-port cascade connection. In: Microwave symposium digest 1981 IEEE MTT-S international, IEEE, pp 507-509

27. Stroud CE. A designers guide to Built-in Self-Test. An overview of BIST, Springer, pp 1-14

28. Sunter S (2004) Circuit and method for determining the location of defect in a circuit. US Patent 6,717,415. https://www.google. com/patents/US6717415

29. Sunter S, Parker K (2009) Testing bridges to nowhere - combining boundary scan and capacitive sensing. In: IEEE international test conference, ITC, 2009. pp 1-10
30. Sunter S, Filliter K, Woo J, McHugh P (2001) A general purpose 1149.4 IC with HF analog test capabilities. In: Proceedings of the IEEE international test conference, 2001. pp 38-45

31. Yao Z, Pan H (2012) Fault diagnosis using magnetic image of PCB. In: IEEE international conference on control (CONTROL), 2012, UKACC. pp 702-707

Abdelghani Renbi was born in 1976 in Khouribga (Morocco), he completed his undergraduate studies in Ecole Supérieure de Technologie of Fes and in Savonia University of Applied Sciences. He received the M.Sc. in Engineering at Jönköping University in 2009. In 2012 he received the degree of Licentiate in Technology and in 2014 the Ph.D. degree, both in Industrial Electronics at Luleå University of Technology. His research interest includes design for testability for state of the art HDIs and power aware electronics. http://www.ltu.se/csee/research/ eislab.

Jerker Delsing was born in 1957 in Umeå (Sweden) and received the M.Sc. in Engineering Physics at Lund Institute of Technology in 1982. In 1985 he received the degree of Licentiate in Technology and in 1988 the Ph.D. degree, both in Electrical Measurement at the Lund Institute of Technology. Early 1995 he was appointed professor in Industrial Electronics at Luleå University of Technology where he is currently working as the scientific head of EISLAB. http://www.ltu.se/ csee/research/eislab. 